TRANSACTIONS OF THE

AMERICAN MATHEMATICAL SOCIETY

Volume 351, Number 7, Pages 2899-2926

S 0002-9947(99)02203-5

Article electronically published on March 10, 1999

\title{
PARTIAL SUBDIFFERENTIALS, DERIVATES AND RADEMACHER'S THEOREM
}

\author{
D. N. BESSIS AND F. H. CLARKE
}

\begin{abstract}
In this paper, we present new partial subdifferentiation formulas in nonsmooth analysis, based upon the study of two directional derivatives. Simple applications of these formulas include a new elementary proof of Rademacher's Theorem in $\mathbb{R}^{n}$, as well as some results on Gâteaux and Fréchet differentiability for locally Lipschitz functions in a separable Hilbert space. RÉsumé. Dans cet article, nous présentons de nouvelles formules de sousdif-

férentiation partielle en analyse nonlisse, basées sur l'étude de deux dérivées directionnelles. Une simple application de ces formules nous permet d'obtenir une nouvelle preuve élémentaire du théorème de Rademacher dans $\mathbb{R}^{n}$, ainsi que certains résultats sur la différentiabilité Gâteaux ou Fréchet des fonctions localement Lipschitz sur un espace de Hilbert séparable.
\end{abstract}

\section{INTRODUCTION}

Throughout this article, let $(H,\|\cdot\|)$ denote a real Hilbert space with inner product $\langle\cdot, \cdot\rangle$. We say that a function $f: H \longrightarrow \mathbb{R}$ is locally Lipschitz if for all $x$ in $H$, there exists $K_{x}>0$ and $\eta_{x}>0$ such that

$$
|f(y)-f(z)| \leq K_{x}\|y-z\| \quad \forall y, z \in x+\eta_{x} B,
$$

where $B$ denotes the open unit ball in $H$. We refer to $K_{x}$ as a Lipschitz constant for $f$ at $x$.

When $H=\mathbb{R}$, locally Lipschitz functions, contrary to merely continuous functions, are known to be differentiable almost everywhere in the sense of linear Lebesgue measure. If $H$ is separable, locally Lipschitz functions are still wellbehaved, since they are differentiable on a dense set of points [21]. We should be careful about the word "differentiable" here, since we can consider at least two different notions of differentiability in $H$, as we now recall.

We say that $f: H \longrightarrow \mathbb{R}$ is Gâteaux differentiable at a point $x$ in $H$, if there exists an element $\xi_{x}$ in $H$ such that

$$
\lim _{t \downarrow 0} \frac{f(x+t v)-f(x)}{t}=\left\langle\xi_{x}, v\right\rangle \quad \forall v \in H .
$$

This equality uniquely determines $\xi_{x}$ which is denoted $f_{G}^{\prime}(x)$ and referred to as the Gâteaux derivative of $f$ at $x$.

Received by the editors February 2, 1997.

1991 Mathematics Subject Classification. Primary 26E99; Secondary 46G05, 49J50, 58B10.

Key words and phrases. Nonsmooth analysis, locally Lipschitz functions, directional derivates, partial subdifferentials, Gâteaux and Fréchet derivatives.

We gratefully acknowledge the support of the Natural Sciences and Engineering Research Council of Canada, and of le Fonds FCAR du Québec. 
Should the convergence in (2) be uniform with respect to $v$ in bounded sets, $f$ is said to have a Fréchet derivative at $x$, denoted $f_{F}^{\prime}(x)$. This is equivalent to requiring

$$
\lim _{h \rightarrow 0} \frac{f(x+h)-f(x)-\left\langle f_{F}^{\prime}(x), h\right\rangle}{\|h\|}=0 .
$$

Besides the obvious case when $H=\mathbb{R}$, Gâteaux and Fréchet derivatives coincide for locally Lipschitz functions in $\mathbb{R}^{n}$. In that case, we will simply say that $f$ has a derivative at $x$, denoted $f^{\prime}(x)$, without ambiguity.

Should $H$ be separable with countable orthonormal basis $\left\{e_{1}, e_{2}, \ldots\right\}$, we denote $x=\left(x_{1}, x_{2}, \ldots\right):=\sum_{i=1}^{\infty} x_{i} e_{i}$ an element of $H$ (with $x_{i}=\left\langle x, e_{i}\right\rangle$ for all $i$ in $\left.\mathbb{N}\right)$. The $i^{\text {th }}$ partial derivative of $f$ at $x$ with respect to the basis $\left\{e_{1}, e_{2}, \ldots\right\}$ is the real number:

$$
\frac{\partial f}{\partial x_{i}}(x):=\lim _{t \rightarrow 0} \frac{g_{i}(t)-g_{i}(0)}{t}, \text { whenever this limit exists, }
$$

where $g_{i}(t):=f\left(x+t e_{i}\right)$ for all $t$ in $\mathbb{R}$. Of course, if $f$ has a Gâteaux derivative (let alone a Fréchet derivative) at $x$, then all partial derivatives exist and we have

$$
f_{G}^{\prime}(x)=\left(\frac{\partial f}{\partial x_{1}}(x), \frac{\partial f}{\partial x_{2}}(x), \ldots\right) \in H .
$$

But even for a locally Lipschitz function $f$ on $\mathbb{R}^{2}$, the converse may not hold; in other words, even if $\left(\frac{\partial f}{\partial x}\left(x_{0}, y_{0}\right), \frac{\partial f}{\partial y}\left(x_{0}, y_{0}\right)\right)$ exists as an element of $\mathbb{R}^{2}$ at some point $\left(x_{0}, y_{0}\right)$, the derivative $f^{\prime}\left(x_{0}, y_{0}\right)$ may not exist, i.e. we may not have the smooth partial differentiation formula

$$
f^{\prime}\left(x_{0}, y_{0}\right)=\left(\frac{\partial f}{\partial x}\left(x_{0}, y_{0}\right), \frac{\partial f}{\partial y}\left(x_{0}, y_{0}\right)\right) .
$$

(To deduce this, one requires in addition, for example, the continuity at the point $\left(x_{0}, y_{0}\right)$ of one of the partial derivatives.) However, if we consider the problem globally, instead of locally, we could wonder how often (6) holds. It cannot hold in generic terms: just consider the function $(x, y) \longmapsto f(x, y)=g(x)$ where $g$ is a locally Lipschitz function on $\mathbb{R}$ that is differentiable only outside a residual set (i.e. a set which contains a countable intersection of dense open sets) of $\mathbb{R}$ (for such a function, see [4]). But Rademacher's Theorem asserts that (6) holds almost everywhere in the sense of plane Lebesgue measure (see [18], [23] and [24]). What is surprising is that, contrary to what happens on the line, not just any set of plane Lebesgue measure zero can be the set of points of non-differentiability of some locally Lipschitz function on $\mathbb{R}^{2}$, as David Preiss showed in [21]. Motivated by these remarks, we try to reformulate Rademacher's conclusion in a different way. For that purpose, we weaken the definition of derivatives and consider subderivatives or subgradients.

We say that an element $\xi$ of $H$ is a D-subgradient (or Dini subgradient or Fréchet subderivative or even weak-Hadamard subderivative [3]) of $f$ at $x$ if

$$
\liminf _{h \rightarrow 0} \frac{f(x+h)-f(x)-\langle\xi, h\rangle}{\|h\|} \geq 0 .
$$

The D-subdifferential of $f$ at $x$ is the (possibly empty) set

$$
\partial_{D} f(x):=\{\xi \in H: \xi \text { satisfies }(7)\} .
$$


The potential advantage of dealing with D-subgradients is that we have inequalities rather than equalities to handle.

If $H$ is separable, using the same notations as above, we define the $i^{\text {th }}$ partial D-subdifferential of $f$ at $x$ with respect to some basis $\left\{e_{1}, e_{2}, \ldots\right\}$ to be

$$
\partial_{D / x_{i}} f(x):=\partial_{D} g_{i}(0),
$$

where $g_{i}(t):=f\left(x+t e_{i}\right)$ for all $t$ in $\mathbb{R}$. A nonsmooth analogue to (6) in $\mathbb{R}^{2}$ would be

$$
\partial_{D} f(x, y)=\partial_{D / x} f(x, y) \times \partial_{D / y} f(x, y),
$$

where we agree that $\emptyset \times A=A \times \emptyset=\emptyset$ for any set $A$ in $H$. It follows easily that $\partial_{D} f(x, y)$ is contained in $\partial_{D / x} f(x, y) \times \partial_{D / y} f(x, y)$, but the opposite inclusion may not be verified at a particular point. For example, consider the locally Lipschitz function $(x, y) \longmapsto f(x, y)=-\min [|x|,|y|]$; then $\partial_{D / x} f(0,0)=\partial_{D / y}(0,0)=\{0\}$, but $\partial_{D} f(0,0)=\emptyset$. However, if we consider, as before, the problem globally instead of locally, we could wonder on how big a set (10) holds. We will see later that this is related to the following issue: if all the partial derivatives of a locally Lipschitz function $f$ exist everywhere on $\mathbb{R}^{2}$, on how big a set can we say that the derivative of $f$ exists (and satisfies (6))? The answer to both questions is a staunch set (see Section 2) which is not only a set of full plane Lebesgue measure (i.e. a set whose complement has plane Lebesgue measure zero) but also a residual set. It is interesting to note that the validity of the nonsmooth partial subdifferentiation formula (10) on a staunch set represents a new union of nonsmooth analysis, measure and category theories and will be the key idea in proving subsequent results on differentiability of locally Lipschitz functions.

The plan of the paper is as follows: in Section 2, we will summarize the tools from nonsmooth analysis and measure theory we will need; in Section 3, we will derive some new nonsmooth partial subdifferentiation formulas [Theorems 3.1 and 3.2 ; Section 4 is devoted to differentiation results in $\mathbb{R}^{n}$, including a new elementary proof of Rademacher's Theorem [Corollary 4.12]; in Section 5, we will address the question of Gâteaux differentiability .

[Theorem 5.1] and intermediate subdifferentiability [Theorem 5.8] and in Section 6 , we will prove some alternative results on Fréchet differentiability [Theorem 6.1 and 6.3] for a locally Lipschitz real-valued function on a separable Hilbert space; finally, in the last section, we will consider special classes of functions such as convex functions, pointwise maxima and distance functions..

\section{Preliminaries}

Besides the notations introduced in Section 1 , we will denote $B(x ; \varrho)$ and $\bar{B}(x ; \varrho)$ respectively the open and closed balls in $H$ centered at $x$ with radius $\varrho>0$, and $\lambda_{n}$ the Lebesgue measure on $\mathbb{R}^{n}$, for $n$ in $\mathbb{N}$. We recall also that a $\lambda_{n}$-null set in $\mathbb{R}^{n}$ is a set of $\lambda_{n}$-measure zero, whereas a $\lambda_{n}$-full set in $\mathbb{R}^{n}$ is the complement of a $\lambda_{n}$-null set. Moreover, the notation " $v_{i} \rightarrow v$ " (or " $v=\lim v_{i}$ ") means that the sequence $\left\{v_{i}\right\}$ converges strongly to $v$, whereas the notation " $v_{i} \rightarrow v$ " (or " $v=\mathrm{w}-\lim v_{i}$ ") means that the sequence $\left\{v_{i}\right\}$ converges weakly to $v$.

- Dini directional derivates: (see [2], [3] and [8]) 
We will use two notions of directional derivates of a function $f$ at a point $x$ of $H$ in the direction $v$ of $H$, defined as follows:

\section{Definition 2.1. Upper and lower classical Dini directional derivates:}

$$
\underline{D} f(x ; v):=\liminf _{t \downarrow 0} \frac{f(x+t v)-f(x)}{t} \text { and } \bar{D} f(x ; v):=\limsup _{t \downarrow 0} \frac{f(x+t v)-f(x)}{t} .
$$

Definition 2.2. Weak Dini directional derivate:

$$
f_{D}^{\prime}(x ; v):=\inf _{\left\{v_{i}\right\} \times\left\{t_{i}\right\}} \liminf _{i \rightarrow \infty} \frac{f\left(x+t_{i} v_{i}\right)-f(x)}{t_{i}},
$$

where the infimum is taken over all sequences $\left\{v_{i}\right\}$ converging weakly to $v$ and over all real positive sequences $\left\{t_{i}\right\}$ decreasing to 0 .

The classical Dini derivates $\underline{D} f$ and $\bar{D} f$ are a direct generalization of Dini's four derived numbers for an arbitrary real-valued function of one variable [25]. The weak Dini derivate $f_{D}^{\prime}$ is another generalization that has the advantage of being directly related to D-subgradients. Indeed, we have

$$
\xi \in \partial_{D} f(x) \Longleftrightarrow\langle\xi, v\rangle \leq f_{D}^{\prime}(x ; v) \forall v \in H .
$$

Classical and weak Dini directional derivates are closely related to Gâteaux and Fréchet derivatives in the following sense:

\section{Proposition 2.1.}

(i) $f_{G}^{\prime}(x)=\xi \Longleftrightarrow\langle\xi, v\rangle \leq \underline{D} f(x ; v)$ and $\langle\xi, v\rangle \geq \bar{D} f(x ; v)$ for all $v$ in $H$.

(ii) $f_{F}^{\prime}(x)$ exists $\Longleftrightarrow \partial_{D} f(x) \neq \emptyset$ and $\partial_{D}(-f)(x) \neq \emptyset$, in which case $\partial_{D} f(x)=-\partial_{D}(-f)(x)=\left\{f_{F}^{\prime}(x)\right\}$.

Classical and weak directional derivates also enjoy nice properties, as is summarized in the following proposition:

\section{Proposition 2.2.}

(a) Positive homogeneity in the direction. $\underline{D} f(x ; \lambda v)=\lambda \underline{D} f(x ; v)$ and $f_{D}^{\prime}(x ; \lambda v)=\lambda f_{D}^{\prime}(x ; v)$ for all $\lambda>0$.

(b) When $H$ is finite-dimensional, we have the simpler characterization:

$$
f_{D}^{\prime}(x ; v)=\liminf _{\substack{v^{\prime} \rightarrow v \\ t \downarrow 0}} \frac{f\left(x+t v^{\prime}\right)-f(x)}{t} .
$$

Moreover, if $f$ is locally Lipschitz, then $f_{D}^{\prime}(x ; v)=\underline{D} f(x ; v)$.

(c) When $f$ is continuous in $H$, then for each fixed $v$ in $H$, the function $x \longmapsto$ $\underline{D} f(x ; v)$ is Borel-measurable, and if $H$ is separable, the function $x \longmapsto f_{D}^{\prime}(x ; v)$ is also Borel-measurable.

(d) When $f$ is locally Lipschitz in $H$ with constant $K_{x}$, then for each fixed $x$ in $H$, $v \longmapsto \underline{D} f(x ; v)$ is a real-valued Lipschitz function with constant $K_{x}$, whereas $v \longmapsto f_{D}^{\prime}(x ; v)$ is either a real-valued Lipschitz function with constant $K_{x}$ or else identically equal to $-\infty$.

- $\left\{\mathbf{e}_{\mathbf{n}}\right\}$-full sets: (see [1], [2], [12], [16] and [20]) 
To prove an extension of Rademacher's Theorem in separable Hilbert spaces, say, one may think of a generalization of the notion of a set of Lebesgue measure zero in $\mathbb{R}^{n}$. It is well known that there is no analogue to Lebesgue measure in infinite-dimensional spaces [12]. However, given any countable orthonormal basis $\left\{e_{1}, e_{2}, \ldots\right\}$ of $H$, it is possible to define a Borel probability measure with satisfying properties on the Hilbert cube of $H$.

We recall that for $a$ in $\mathbb{N}$, the $a^{t h}$ Hilbert cube associated with $\left\{e_{1}, e_{2}, \ldots\right\}$ is the following set:

$$
K^{a}:=\left\{x \in H:\left|\left\langle x, e_{k}\right\rangle\right| \leq \frac{a}{k} \forall k=1,2, \ldots\right\} \simeq \prod_{k=1}^{\infty}\left[-\frac{a}{k} ; \frac{a}{k}\right] .
$$

Let us endow each interval $[-a / k ; a / k]$ with the Borel probability measure $\mu_{a}^{(k)}:=$ $(k / 2 a) \lambda_{1} \quad(k=1,2, \ldots)$ and each cube $K_{n}^{a}:=\prod_{k=1}^{n}[-a / k ; a / k]$ with the Borel product probability measure $\mu_{n}^{a}:=\mu_{a}^{(1)} \otimes \ldots \otimes \mu_{a}^{(n)}(n=1,2, \ldots)$. Then we know there exists a Borel probability measure $\nu^{a}$ on $K^{a}$ such that if $p_{n}^{a}$ denotes the projection of $K^{a}$ onto $K_{n}^{a}$, then $\mu_{n}^{a}$ is the image of $\nu^{a}$ by $p_{n}^{a}$. The measure $\nu^{a}$ has the following properties:

Proposition 2.3. Let $\left\{e_{1}, e_{2}, \ldots\right\}$ be a countable orthonormal basis of $H$.

(i) If $A$ is a Borel set in $H$, then $A \cap K^{a}$ is $\nu^{a}$-measurable.

(ii) If $x_{0}$ is in $K^{a}$ and if $r>0$, then $\nu^{a}\left(B\left(x_{0} ; r\right) \cap K^{a}\right)>0$.

(iii) For each $n$ in $\mathbb{N}$, if $\tilde{K}_{n}^{a}:=\prod_{k>n}[-a / k ; a / k]$ and if $\tilde{\mu}_{n}^{a}$ is the Borel probability measure on $\tilde{K}_{n}^{a}$ (constructed as above), then $\nu^{a}=\mu_{n}^{a} \otimes \tilde{\mu}_{n}^{a}$.

We now make a new definition:

Definition 2.3. Given a countable orthonormal basis $\left\{e_{1}, e_{2}, \ldots\right\}$ of $H$, a set $A$ in $H$ is said to be a $\left\{\mathbf{e}_{\mathbf{n}}\right\}$-full set if $A$ is a Borel set of $H$ and if

$$
\nu^{a}\left(A \cap K^{a}\right)=1 \text { for all } a \text { in } \mathbb{N} .
$$

Here are some interesting facts about $\left\{e_{n}\right\}$-full sets:

Proposition 2.4. Let $\left\{e_{1}, e_{2}, \ldots\right\}$ be a countable orthonormal basis of $H$.

(a) Every $\left\{e_{n}\right\}$-full set is uncountable.

(b) Every $\left\{e_{n}\right\}$-full set is dense.

(c) Every countable intersection of $\left\{e_{n}\right\}$-full sets is a $\left\{e_{n}\right\}$-full set.

(d) When $H=\mathbb{R}^{n},\left\{e_{n}\right\}$-full sets are merely Borel sets of full $\lambda_{n}$-measure.

- Staunch sets: (see [10], [15], [28] and [29])

The notion of porosity was first introduced by Dolženko [10]. We recall Zajǐcek's metric definition of porosity:

Definition 2.4. Let $C$ be an arbitrary subset of $H$.

A point $y_{0}$ in $H$ is a porosity point of $C$ if

$$
\limsup _{r \downarrow 0} \frac{\left.\gamma\left[B\left(y_{0} ; r\right), C\right)\right]}{r}>0,
$$

where $\left.\gamma\left[B\left(y_{0} ; r\right), C\right)\right]$ is defined for all $r>0$ as the following real number:

$$
\sup \left\{r^{\prime}>0 \text { : for some } z \text { in } H, B\left(z ; r^{\prime}\right) \subseteq B\left(y_{0} ; r\right) \text { and } B\left(z ; r^{\prime}\right) \cap C=\emptyset\right\} \text {. }
$$


(By convention, $\sup \emptyset=0$ in this definition.)

In particular, a point $y_{0}$ in $H$ is a porosity point of $C$ if there exist a real number $p \in(0,1)$, a sequence $\left\{v_{n}\right\}$ in $H$ with $\left\|v_{n}\right\|=1$ for all $n$ in $\mathbb{N}$, and two real positive sequences $\left\{t_{n}\right\}$ and $\left\{r_{n}\right\}$ decreasing to 0 such that:

$$
B\left(y_{0}+t_{n} v_{n} ; p r_{n}\right) \subseteq B\left(y_{0} ; r_{n}\right) \cap C^{c} \text { for all } n \text { in } \mathbb{N} \text {. }
$$

Definition 2.5. A set $C$ in $H$ is said to be porous if all its points are porosity points of $C$. A set $S$ in $H$ is said to be $\sigma$-porous if it is a countable union of porous sets.

We are now in a position to make a new definition that we will use often hereafter:

Definition 2.6. Let $U$ be an open set in $H$. A set $S$ in $U$ is said to be staunch in $U$ if its complement with respect to $U$ is $\sigma$-porous.

Let us now recall some notions about category in a Hilbert space $H$ (see [14] and [25]). A set in $H$ is said to be dense if its closure is the whole space. A set in $H$ is said to be rare if the interior of its closure is empty. A countable union of rare sets is said to be of first category. The complement of a set of first category (i.e. a set which contains a countable intersection of dense open sets) is called a residual set. Baire's Theorem implies in particular that any residual set of $H$ is dense and uncountable in $H$.

\section{Proposition 2.5.}

(a) Every porous set in $H$ is meager.

(b) Every $\sigma$-porous set in $H$ is of first category.

In $\mathbb{R}^{n}, \sigma$-porous sets are all the more interesting since they are negligible in the sense of both category and measure:

Proposition 2.6. Porous and $\sigma$-porous sets in $\mathbb{R}^{n}$ are $\lambda_{n}$-null sets.

Remark 2.1. By Propositions 2.5 and 2.6, we see that staunch sets in $H$ are residual sets, and staunch sets in $\mathbb{R}^{n}$ are $\lambda_{n}$-full sets. Moreover, a countable intersection of staunch sets is itself staunch.

Remark 2.2. There exist residual sets of full $\lambda_{n}$-measure in $\mathbb{R}^{n}$ that are not staunch sets, as Zaj̃̄icek showed in [29].

\section{Nonsmooth Partial Subdifferentiation Formulas}

The following two theorems are new. The first one deals with $\underline{D} f(x ; v)$ while the other one is a corresponding version with $f_{D}^{\prime}(x ; v)$.

Theorem 3.1. Let $H$ and $H^{\prime}$ be two real separable nontrivial Hilbert spaces, let $U$ and $V$ be nonempty open subsets of $H$ and $H^{\prime}$ respectively, and let $f: U \times V \rightarrow \mathbb{R}$ be locally Lipschitz on $U \times V$.

Then there exists a staunch Borel subset $S$ of $U \times V$ such that for any $x$ in $U$, the set $\{y \in V:(x, y) \in S\}$ is staunch in $V$, and such that for each $(x, y)$ in $S$, we have:

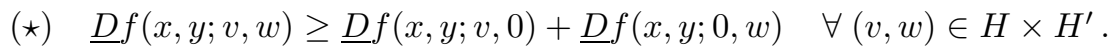

Theorem 3.2. Let $H$ be a real separable nontrivial Hilbert space, let $U$ and $V_{n}$ be nonempty open subsets of $H$ and $\mathbb{R}^{n}$ (with $n \geq 1$ ) respectively, and let $f: U \times V_{n} \rightarrow$ $\mathbb{R}$ be locally Lipschitz on $U \times V_{n}$. 
Then there exists a staunch Borel subset $S$ of $U \times V_{n}$ such that for any $x$ in $U$, the set $\left\{y \in V_{n}:(x, y) \in S\right\}$ is staunch in $V_{n}$, and such that for each $(x, y)$ in $S$, we have:

$(\star \star) \quad f_{D}^{\prime}(x, y ; v, w) \geq f_{D}^{\prime}(x, y ; v, 0)+\underline{D} f(x, y ; 0, w) \quad \forall(v, w) \in H \times \mathbb{R}^{n}$.

Proof of Theorem 3.1. The Borel character of the set $\{(x, y) \in U \times V:(\star)$ is true $\}$ is easy to see (using the separability of $H$ and $H^{\prime}$ and Proposition 2.2).

We will prove $(\star)$ only for any $(v, w)$ in $H \times S(0 ; 1) \equiv H \times\left\{w \in H^{\prime}:\|w\|_{H^{\prime}}=1\right\}$, since $(\star)$ is satisfied for $w=0$ and $\underline{D} f(x, y ; v, w)$ is positively homogeneous in $(v, w)$. We will denote $\mathbb{Q}_{+}^{*}$ the set of all positive rational numbers.

Part A. Let $x$ be given in $U$. Consider the set $S_{x}:=\{y \in V:(\star)$ is false $\}$. We proceed to show that $S_{x}$ is $\sigma$-porous. For that purpose, let $D$ and $D^{\prime}$ be dense and countable sets in $H$ and $S(0 ; 1)$ respectively, and denote

$$
\begin{aligned}
C(v, w, r, \varepsilon):=\left\{y \in S_{x}: \frac{f(x+t v, y)-f(x, y)}{t}>r>\underline{D} f(x, y ; v, w)\right. \\
\quad-\underline{D} f(x, y ; 0, w) \text { for all } t \text { in }(0, \varepsilon)\} .
\end{aligned}
$$

Let us show that $S_{x}=\bigcup_{(v, w, r, \varepsilon) \in D \times D^{\prime} \times \mathbb{Q} \times \mathbb{Q}_{+}^{*}} C(v, w, r, \varepsilon)$.

One inclusion is obvious. For the opposite one, if $y$ belongs to $S_{x}$, there exists $(\tilde{v}, \tilde{w})$ in $H \times S(0 ; 1)$ such that

$$
\underline{D} f(x, y ; \tilde{v}, \tilde{w})<\underline{D} f(x, y ; \tilde{v}, 0)+\underline{D} f(x, y ; 0, \tilde{w}) .
$$

The function $f$ being Lipschitz with constant $K_{x, y}>0$ near $(x, y)$, the function $(v, w) \longmapsto \underline{D} f(x, y ; v, w)$ is itself Lipschitz with constant $K_{x, y}>0$ and hence finite. Thus we can find $r$ in $\mathbb{Q}$ such that

$$
\underline{D} f(x, y ; \tilde{v}, 0)>r>\underline{D} f(x, y ; \tilde{v}, \tilde{w})-\underline{D} f(x, y ; 0, \tilde{w}),
$$

and we can pick $(v, w)$ in $D \times D^{\prime}$ close enough to $(\tilde{v}, \tilde{w})$ so that

$$
\underline{D} f(x, y ; v, 0)>r>\underline{D} f(x, y ; v, w)-\underline{D} f(x, y ; 0, w)
$$

[this is possible because inequalities are strict and $\underline{D} f\left(x, y ; u_{1}, u_{2}\right)$ and $\underline{D} f\left(x, y ; \tilde{u}_{1}, \tilde{u}_{2}\right)$ differ by at most $K_{x, y}\left\|\left(u_{1}, u_{2}\right)-\left(\tilde{u}_{1}, \tilde{u}_{2}\right)\right\|$ in absolute terms].

Finally, by definition of $\underline{D} f(x, y ; v, 0)$, there exists $\varepsilon$ in $\mathbb{Q}_{+}^{*}$ such that:

$$
\frac{f(x+t v, y)-f(x, y)}{t}>r \quad \forall t \in(0, \varepsilon)
$$

(and such that $x+(0, \varepsilon) v$ is a subset of the open set $U$ ). Hence $y$ belongs to $C(v, w, r, \varepsilon)$ for some $(v, w, r, \varepsilon)$ in $D \times D^{\prime} \times \mathbb{Q} \times \mathbb{Q}_{+}^{*}$.

Since $S_{x}$ can be written as a countable union of subsets of $V$, we only need to show that these subsets are porous to obtain the desired result. To simplify, denote $C \equiv C(v, w, r, \varepsilon)$. If $C=\emptyset$, then $C$ is porous a fortiori. If not, let us show that any $y_{0}$ in $C$ is a porosity point of $C$. Let $K_{0}>0$ be a Lipschitz constant of $f$ on $\left(x, y_{0}\right)+\eta_{0} B \subseteq U \times V$, where $\eta_{0}>0$ and $B$ is the open unit ball in $H \times H^{\prime}$. Since $y_{0}$ is in $C$, we have in particular:

$$
r>\underline{D} f\left(x, y_{0} ; v, w\right)-\underline{D} f\left(x, y_{0} ; 0, w\right) .
$$

Let us pick $0<\delta<4 K_{0}$ such that $r>\delta+\underline{D} f\left(x, y_{0} ; v, w\right)-\underline{D} f\left(x, y_{0} ; 0, w\right)$ and let us pick $\left\{t_{i}\right\} \downarrow 0$ such that:

$$
\lim _{i \rightarrow \infty} \frac{f\left(x+t_{i} v, y_{0}+t_{i} w\right)-f\left(x, y_{0}\right)}{t_{i}}=\underline{D} f\left(x, y_{0} ; v, w\right) .
$$


Of course, we have

$$
\begin{aligned}
\underline{D} f\left(x, y_{0} ; 0, w\right) & \leq \liminf _{i \rightarrow \infty} \frac{f\left(x, y_{0}+t_{i} w\right)-f\left(x, y_{0}\right)}{t_{i}} \\
& =\lim _{k \rightarrow \infty} \frac{f\left(x, y_{0}+t_{i_{k}} w\right)-f\left(x, y_{0}\right)}{t_{i_{k}}},
\end{aligned}
$$

taking a subsequence if necessary. Thus, we obtain:

$$
\begin{aligned}
r & >\delta+\underline{D} f\left(x, y_{0} ; v, w\right)-\underline{D} f\left(x, y_{0} ; 0, w\right) \\
& \geq \delta+\lim _{k \rightarrow \infty}\left[\frac{f\left(x+t_{i_{k}} v, y_{0}+t_{i_{k}} w\right)-f\left(x, y_{0}\right)}{t_{i_{k}}}\right]-\lim _{k \rightarrow \infty}\left[\frac{f\left(x, y_{0}+t_{i_{k}} w\right)-f\left(x, y_{0}\right)}{t_{i_{k}}}\right] \\
& =\delta+\lim _{k \rightarrow \infty}\left[\frac{f\left(x+t_{i_{k}} v, y_{0}+t_{i_{k}} w\right)-f\left(x, y_{0}+t_{i_{k}} w\right)}{t_{i_{k}}}\right] .
\end{aligned}
$$

Now let us pick $k_{0}$ in $\mathbb{N}$ such that if $k \geq k_{0}$, we have:

$$
\begin{aligned}
& \text { (1) } r>\frac{\delta}{2}+\frac{f\left(x+t_{i_{k}} v, y_{0}+t_{i_{k}} w\right)-f\left(x, y_{0}+t_{i_{k}} w\right)}{t_{i_{k}}} \quad \text { (by definition of limit), } \\
& \text { (2) } 0<t_{i_{k}}<\varepsilon \quad \text { (possible since } t_{i_{k}} \downarrow 0 \text { ), } \\
& \text { (3) } 0<t_{i_{k}}<\min \left(\frac{\eta_{0}}{4} ; \frac{\eta_{0}}{2(\|v\|+1)}\right) \quad \text { (idem). }
\end{aligned}
$$

Relabel $\left\{t_{i_{k}}\right\}_{k \geq k_{0}} \equiv\left\{t_{j}\right\}_{j=1}^{\infty}$. And let us look at all points $y$ in $C \cap B\left(y_{0} ; \frac{\eta_{0}}{2}\right)$. In particular, we have: $\frac{f(x+t v, y)-f(x, y)}{t}>r$ if $0<t<\varepsilon$. Hence by (2), for every $j=1,2, \ldots$, we have:

$$
\frac{f\left(x+t_{j} v, y\right)-f(x, y)}{t_{j}}>r .
$$

Combining with (1), we obtain after simplification, for each $j=1,2, \ldots$,

$$
\frac{f\left(x+t_{j} v, y\right)-f\left(x+t_{j} v, y_{0}+t_{j} w\right)}{t_{j}}+\frac{f\left(x, y_{0}+t_{j} w\right)-f(x, y)}{t_{j}}>\frac{\delta}{2} .
$$

Now since each of the preceding arguments of $f$ are points in $\left(x, y_{0}\right)+\eta_{0} B$ [by condition (3) and because $\left\|y-y_{0}\right\|<\frac{\eta_{0}}{2}$ and $\left.\|w\|=1\right]$, we get:

$$
\begin{gathered}
\frac{\delta}{2}<K_{0} \frac{\left\|y-\left(y_{0}+t_{j} w\right)\right\|}{t_{j}}+K_{0} \frac{\left\|y-\left(y_{0}+t_{j} w\right)\right\|}{t_{j}}, \quad \forall j=1,2, \ldots, \\
\text { i.e. } \quad\left\|y-\left(y_{0}+t_{j} w\right)\right\|>\frac{\delta}{4 K_{0}} t_{j}, \quad \forall j=1,2, \ldots .
\end{gathered}
$$

Hence we proved that for each $j=1,2, \ldots$, the open ball $B\left(y_{0}+t_{j} w ; \frac{\delta}{4 K_{0}} t_{j}\right)$ does not intersect $C \cap B\left(y_{0} ; \frac{\eta_{0}}{2}\right)$. Now since for each $j=1,2, \ldots, B\left(y_{0}+t_{j} w ; \frac{\delta}{4 K_{0}} t_{j}\right)$ is also contained in $B\left(y_{0} ; 2 t_{j}\right)$ [because $0<\delta<4 K_{0}$ and $\|w\|=1$ ] which is itself contained in the bigger ball $B\left(y_{0} ; \frac{\eta_{0}}{2}\right)$ by condition (3), it is then obvious to see that $y_{0}$ is a porosity point of $C$.

Part B. To prove now that the set where $(\star)$ is satisfied is a staunch set in $U \times V$, denote: $S:=\{(x, y) \in U \times V:(\star)$ is false $\}$ and

$$
\begin{aligned}
C(v, w, r, \varepsilon):=\left\{(x, y) \in S: \frac{f(x+t v, y)-f(x, y)}{t}>r>\underline{D} f(x, y ; v, w)\right. \\
\quad-\underline{D} f(x, y ; 0, w) \text { for all } t \text { in }(0, \varepsilon)\} .
\end{aligned}
$$

Then we easily show, as before, that $S=\bigcup_{(v, w, r, \varepsilon) \in D \times D^{\prime} \times \mathbb{Q} \times \mathbb{Q}_{+}^{*}} C(v, w, r, \varepsilon)$.

It remains to show that each $C \equiv C(v, w, r, \varepsilon)$ is porous. Let $\left(x_{0}, y_{0}\right)$ be a point in $C$, and denote by $K_{0}>0$ a Lipschitz constant of $f$ on $\left(x_{0}, y_{0}\right)+\eta_{0} B \subseteq U \times V$, 
where $\eta_{0}>0$ and $B$ is the open unit ball in $H \times H^{\prime}$. As before, we can find a real positive sequence $\left\{t_{j}\right\}$ decreasing to 0 , such that:

(1) $r>\frac{\delta}{2}+\frac{f\left(x_{0}+t_{j} v, y_{0}+t_{j} w\right)-f\left(x_{0}, y_{0}+t_{j} w\right)}{t_{j}}$,

(2) $0<t_{j}<\varepsilon$,

(3) $0<t_{j}<\min \left(\frac{\eta_{0}}{8} ; \frac{\eta_{0}}{4(\|v\|+1)}\right)$.

Now let us look at all points $(x, y)$ in $C \cap B\left(\left(x_{0}, y_{0}\right) ; \frac{\eta_{0}}{4}\right)$. We therefore have by (2), for each $j=1,2, \ldots$ :

$$
\frac{f\left(x+t_{j} v, y\right)-f(x, y)}{t_{j}}>r
$$

Combining with (1), we obtain after simplification, for each $j=1,2, \ldots$,

$$
\frac{f\left(x+t_{j} v, y\right)-f\left(x_{0}+t_{j} v, y_{0}+t_{j} w\right)}{t_{j}}+\frac{f\left(x_{0}, y_{0}+t_{j} w\right)-f(x, y)}{t_{j}}>\frac{\delta}{2} .
$$

Now since each of the preceding arguments of $f$ are points in $\left(x_{0}, y_{0}\right)+\eta_{0} B$ [by condition (3) and because $\left.\left\|(x, y)-\left(x_{0}, y_{0}\right)\right\|_{H \times H^{\prime}}<\frac{\eta_{0}}{4}\right]$, we get for all $j=1,2, \ldots$ :

$$
\frac{\delta}{2}<K_{0} \frac{\sqrt{\left\|x-x_{0}\right\|_{H}^{2}+\left\|y-\left(y_{0}+t_{j} w\right)\right\|_{H^{\prime}}^{2}}}{t_{j}}+K_{0} \frac{\sqrt{\left\|x-x_{0}\right\|_{H}^{2}+\left\|y-\left(y_{0}+t_{j} w\right)\right\|_{H^{\prime}}^{2}}}{t_{j}}
$$

i.e.

$$
\left\|(x, y)-\left(x_{0}, y_{0}+t_{j} w\right)\right\|_{H \times H^{\prime}}>\frac{\delta}{4 K_{0}} t_{j}, \quad \forall j=1,2, \ldots .
$$

Hence we proved that for each $j=1,2, \ldots$, the open ball $B\left(\left(x_{0}, y_{0}+t_{j} w\right) ; \frac{\delta}{4 K_{0}} t_{j}\right)$ does not intersect $C \cap B\left(\left(x_{0}, y_{0}\right) ; \frac{\eta_{0}}{4}\right)$. Now since for each $j=1,2, \ldots$, $B\left(\left(x_{0}, y_{0}+t_{j} w\right) ; \frac{\delta}{4 K_{0}} t_{j}\right)$ is also contained in $B\left(\left(x_{0}, y_{0}\right) ; 2 t_{j}\right)$ [because $0<\delta<$ $4 K_{0}$ and $\left.\|w\|=1\right]$ which is itself contained in the bigger ball $B\left(\left(x_{0}, y_{0}\right) ; \frac{\eta_{0}}{4}\right)$ by condition (3), it is then obvious to see that $\left(x_{0}, y_{0}\right)$ is a porosity point of $C$.

Remark 3.1. The set $C$ in the proof of Part A of Theorem 3.1 actually satisfies the following stronger property: there exists $w$ in $H \backslash\{0\}$ such that for any $y_{0}$ in $C$, we can exhibit $0<p<1$ and two real positive sequences $\left\{t_{n}\right\}$ and $\left\{r_{n}\right\}$ decreasing to 0 such that

$$
B\left(y_{0}+t_{n} w ; p r_{n}\right) \subseteq B\left(y_{0} ; r_{n}\right) \cap C^{c} \text { for all } n \text { in } \mathbb{N} .
$$

Such a set is called uniformly directionally porous [28]. In this article, we could define a staunch set to be the complement of a countable union of uniformly directionally porous set and all our results will be valid with this new definition.

Proof of Theorem 3.2. When $H$ is finite-dimensional, $(\star \star)$ is the same as $(\star)$, and we are done. So let us assume $H$ to be infinite-dimensional.

Let us remark that since $f$ is locally Lipschitz as a function of the second variable on $V_{n}$, we have for any $(x, y)$ in $U \times V_{n}$ and any $(v, w)$ in $H \times \mathbb{R}^{n}$ :

$$
f_{D}^{\prime}(x, y ; v, w)=\inf _{\substack{\left\{v_{i}\right\} \\\left\{t_{i}\right\} \downarrow 0}} \liminf _{i \rightarrow+\infty} \frac{f\left(x+t_{i} v_{i}, y+t_{i} w\right)-f(x, y)}{t_{i}},
$$

where the infimum is taken over all sequences $\left\{v_{i}\right\}$ converging weakly to $v$ and over all real positive sequences $\left\{t_{i}\right\}$ decreasing to 0 . Moreover, since $H$ is separable, 
there exists a metric, say $d_{M}$, which induces the weak topology on the (strongly) closed ball $\bar{B}(0 ; M)$. In that case, diagonalization can be applied and we have:

$$
\inf _{\substack{\left\{v_{i}\right\} \times\left\{t_{i}\right\} \\\left\{v_{i}\right\} \subseteq \bar{B}(0, M)}} \liminf _{i \rightarrow \infty} \frac{f\left(x+t_{i} v_{i}\right)-f(x)}{t_{i}}=\liminf _{\substack{v^{\prime} \\ v_{M} \downarrow 0}} \frac{f\left(x+t v^{\prime}\right)-f(x)}{t},
$$

where the infimum is taken over all sequences $\left\{v_{i}\right\}$ in $\left(\bar{B}(0 ; M), d_{M}\right)$ converging to $v$ and over all real positive sequences $\left\{t_{i}\right\}$ decreasing to 0 , and where the notation " $v^{\prime} \stackrel{d_{M}}{\longrightarrow} v$ " means " $v^{\prime}$ is in $\bar{B}(0 ; M)$ and $d_{M}\left(v^{\prime}, v\right) \rightarrow 0$ ". The Borel character of the set $\left\{(x, y) \in U \times V_{n}:(\star \star)\right.$ is true $\}$ is easy to see (using the separability of $H$ and Proposition 2.2). We will prove ( $\star \star)$ only for any $(v, w)$ in $H \times S_{n}(0 ; 1) \equiv H \times\{w \in$ $\left.\mathbb{R}^{n}:\|w\|_{\mathbb{R}^{n}}=1\right\}$, since $(\star \star)$ is satisfied for $w=0$ and $f_{D}^{\prime}(x, y ; v, w)$ is positively homogeneous in $(v, w)$.

As before, the set of all positive rational numbers is denoted by $\mathbb{Q}_{+}^{*}$.

Part A. Let $x$ be given in $U$. Consider the set $S_{x}:=\left\{y \in V_{n}:(\star \star)\right.$ is false $\}$. We proceed to show that $S_{x}$ is $\sigma$-porous. For that purpose, let $D$ and $D^{\prime}$ be dense and countable sets in $H$ and $S_{n}(0 ; 1)$ respectively, let $d_{M}$ be the metric only defined on the (strongly) closed ball $\bar{B}(0 ; M)$ of $H$ which induces the weak topology and denote

$C(v, w, r, M, \varepsilon):=\left\{y \in S_{x}:(\bullet)\right.$ is true for all $t$ in $(0, \varepsilon)$ and $v^{\prime}$ with $\left.d_{M}\left(v^{\prime}, v\right)<\varepsilon\right\}$

where $(\bullet)$ is the following:

$$
\begin{aligned}
& \frac{f\left(x+t v^{\prime}, y\right)-f(x, y)}{t}>r>\inf _{\substack{\left\{v_{i}\right\} \stackrel{i}{\sim} v \\
v_{i} \in \dot{B}(0 ; M) \\
\left\{t_{i}\right\} \downarrow j}} \liminf _{i \rightarrow+\infty} \frac{f\left(x+t_{i} v_{i}, y+t_{i} w\right)-f(x, y)}{t_{i}} \\
& \ldots-\underline{D} f(x, y ; 0, w) .
\end{aligned}
$$

Let us show that $S_{x}=\bigcup_{(v, w, r, M, \varepsilon) \in D \times D^{\prime} \times \mathbb{Q} \times \mathbb{N} \times \mathbb{Q}_{+}^{*}} C(v, w, r, M, \varepsilon)$.

One inclusion is obvious. For the opposite one, if $y$ is in $S_{x}$, there exists $(\tilde{v}, \tilde{w})$ in $H \times S_{n}(0 ; 1)$ such that

$$
f_{D}^{\prime}(x, y ; \tilde{v}, \tilde{w})<f_{D}^{\prime}(x, y ; \tilde{v}, 0)+\underline{D f}(x, y ; 0, \tilde{w}) .
$$

We notice that necessarily $f_{D}^{\prime}(x, y ; \cdot, \cdot) \not \equiv-\infty$; hence $(v, w) \longmapsto f_{D}^{\prime}(x, y ; v, w)$ is a real-valued Lipschitz function. Thus we can find $r$ in $\mathbb{Q}$ such that

$$
f_{D}^{\prime}(x, y ; \tilde{v}, 0)>r>f_{D}^{\prime}(x, y ; \tilde{v}, \tilde{w})-\underline{D} f(x, y ; 0, \tilde{w}),
$$

and we can pick $(v, w)$ in $D \times D^{\prime}$ close enough to $(\tilde{v}, \tilde{w})$ so that

$$
f_{D}^{\prime}(x, y ; v, 0)>r>f_{D}^{\prime}(x, y ; v, w)-\underline{D} f(x, y ; 0, w) .
$$

In particular, we have $f_{D}^{\prime}(x, y ; v, w)<\alpha$, where $\alpha:=r+\underline{D} f(x, y ; 0, w) \in \mathbb{R}$. Hence by definition of $f_{D}^{\prime}$, there exist a sequence $\left\{v_{i}\right\}$ converging weakly to $v$ and a real positive sequence $\left\{t_{i}\right\}$ decreasing to 0 such that:

$$
\liminf _{i \rightarrow+\infty} \frac{f\left(x+t_{i} v_{i}, y+t_{i} w\right)-f(x, y)}{t_{i}}<\alpha .
$$


But, since $v_{i} \stackrel{i}{\rightarrow} v$, there exists $M$ in $\mathbb{N}$ such that $\|v\| \leq \liminf _{i \rightarrow+\infty}\left\|v_{i}\right\| \leq M$; hence

$$
\inf _{\substack{\left\{v_{i}\right\} \\ v_{i} \in \bar{i}(0 ; M) \\\left\{t_{i}\right\} \downarrow 0}} \liminf _{i \rightarrow+\infty} \frac{f\left(x+t_{i} v_{i}, y+t_{i} w\right)-f(x, y)}{t_{i}}<\alpha .
$$

Of course, we have:

$$
\inf _{\substack{\left\{v_{i}\right\} \\ v_{i} \in \bar{B}(0 ; M) \\\left\{t_{i}\right\} \downarrow 0}} \liminf _{i \rightarrow+\infty} \frac{f\left(x+t_{i} v_{i}, y\right)-f(x, y)}{t_{i}} \geq f_{D}^{\prime}(x, y ; v, 0)>r .
$$

Combining the last two displayed inequalities, we therefore have found $(v, w, r, M)$ in $D \times D^{\prime} \times \mathbb{Q} \times \mathbb{N}$ such that:

$$
\begin{aligned}
& \inf _{\left\{v_{i}\right\} \stackrel{i}{\sim} v} \liminf _{i \rightarrow+\infty} \frac{f\left(x+t_{i} v_{i}, y\right)-f(x, y)}{t_{i}}>r>\ldots \\
& v_{i} \in \bar{B}(0 ; M) \\
& \left\{t_{i}\right\} \downarrow 0 \\
& \ldots \inf _{\substack{\left\{v_{i}\right\} \\
v_{i} \in B(0 ; M) \\
\left\{t_{i}\right\} \downarrow 0}} \liminf _{i \rightarrow+\infty} \frac{f\left(x+t_{i} v_{i}, y+t_{i} w\right)-f(x, y)}{t_{i}}-\underline{D} f(x, y ; 0, w) .
\end{aligned}
$$

Finally, since

$$
\inf _{\substack{\left\{v_{i}\right\} \stackrel{i}{\vec{i}} v \\ v_{i} \in \bar{B}(0 ; M) \\\left\{t_{i}\right\} \downarrow \rightarrow \infty}} \liminf _{i \rightarrow+\infty} \frac{f\left(x+t_{i} v_{i}, y\right)-f(x, y)}{t_{i}}=\liminf _{\substack{v^{\prime} \\ t \downarrow 0}} \frac{f\left(x+t v^{\prime}, y\right)-f(x, y)}{t},
$$

we can find $\varepsilon$ in $\mathbb{Q}_{+}^{*}$ such that for any $t$ in $(0, \varepsilon)$ and for any $v^{\prime}$ such that $d_{M}\left(v^{\prime}, v\right)<$ $\varepsilon$

$$
\frac{f\left(x+t v^{\prime}, y\right)-f(x, y)}{t}>r,
$$

which proves that $y$ belongs to $C(v, w, r, M, \varepsilon)$.

Since $S_{x}$ can be written as a countable union of subsets of $V_{n}$, we only need to show that these subsets are porous. To simplify, denote $C \equiv C(v, w, r, M, \varepsilon)$. If $C=\emptyset$, then $C$ is porous a fortiori. If not, let us show that any $y_{0}$ in $C$ is a porosity point of $C$. Let $K_{0}>0$ be a Lipschitz constant of $f$ on $\left(x, y_{0}\right)+\eta_{0} B \subseteq U \times V_{n}$, where $\eta_{0}>0$ and $B$ is the open unit ball in $H \times \mathbb{R}^{n}$. Since $y_{0}$ is in $C$, we have in particular:

$$
r>\inf _{\substack{\left\{v_{i}\right\} \\ v_{i} \in \underset{B}{B}(0 ; M) \\\left\{t_{i}\right\} \downarrow}} \liminf _{i \rightarrow+\infty} \frac{f\left(x+t_{i} v_{i}, y_{0}+t_{i} w\right)-f\left(x, y_{0}\right)}{t_{i}}-\underline{D} f\left(x, y_{0} ; 0, w\right) .
$$

Let us pick $0<\delta<4 K_{0}$ such that:

$$
r>\delta+\inf _{\substack{\left\{v_{i}\right\} \stackrel{i}{\sim} v \\ v_{i} \in \bar{B}(0 ; M) \\\left\{t_{i}\right\} \downarrow 0}} \liminf _{i \rightarrow+\infty} \frac{f\left(x+t_{i} v_{i}, y_{0}+t_{i} w\right)-f\left(x, y_{0}\right)}{t_{i}}-\underline{D f} f\left(x, y_{0} ; 0, w\right) .
$$


Defining $\beta:=r-\delta+\underline{D} f\left(x, y_{0} ; 0, w\right) \in \mathbb{R}$, since

$$
\inf _{\substack{\left\{v_{i}\right\} \stackrel{i}{\rightarrow} v \\ v_{i} \in{ }^{B}(0 ; M) \\\left\{t_{i}\right\} \downarrow 0}} \liminf _{i \rightarrow+\infty} \frac{f\left(x+t_{i} v_{i}, y_{0}+t_{i} w\right)-f\left(x, y_{0}\right)}{t_{i}}<\beta,
$$

there exist a sequence $\left\{v_{i}\right\}$ in $\bar{B}(0 ; M)$ converging weakly to $v$ and a real positive sequence $\left\{t_{i}\right\}$ decreasing to 0 such that:

$\beta>\liminf _{i \rightarrow+\infty} \frac{f\left(x+t_{i} v_{i}, y_{0}+t_{i} w\right)-f\left(x, y_{0}\right)}{t_{i}}=\lim _{k \rightarrow+\infty} \frac{f\left(x+t_{i_{k}} v_{i_{k}}, y_{0}+t_{i_{k}} w\right)-f\left(x, y_{0}\right)}{t_{i_{k}}}$,

taking a subsequence if necessary. Of course, we have:

$$
\begin{aligned}
\underline{D f}\left(x, y_{0} ; 0, w\right) & \leq \liminf _{k \rightarrow+\infty} \frac{f\left(x, y_{0}+t_{i_{k}} w\right)-f\left(x, y_{0}\right)}{t_{i_{k}}} \\
& =\lim _{j \rightarrow+\infty} \frac{f\left(x, y_{0}+t_{i_{k_{j}}} w\right)-f\left(x, y_{0}\right)}{t_{i_{k_{j}}}},
\end{aligned}
$$

taking another subsequence if necessary. Thus we obtain:

$$
\begin{aligned}
r & >\delta+\lim _{j \rightarrow+\infty} \frac{f\left(x+t_{i_{k_{j}}} v_{i_{k_{j}}}, y_{0}+t_{i_{k_{j}}} w\right)-f\left(x, y_{0}\right)}{t_{i_{k_{j}}}}-\lim _{j \rightarrow+\infty} \frac{f\left(x, y_{0}+t_{i_{k_{j}}} w\right)-f\left(x, y_{0}\right)}{t_{i_{k_{j}}}} \\
& =\delta+\lim _{j \rightarrow+\infty} \frac{f\left(x+t_{i_{k_{j}}} v_{i_{k_{j}}}, y_{0}+t_{i_{k_{j}}} w\right)-f\left(x, y_{0}+t_{i_{k_{j}}} w\right)}{t_{i_{k_{j}}}} .
\end{aligned}
$$

Now let us take $j_{0}$ in $\mathbb{N}$ such that if $j \geq j_{0}$, we have:

(1) $r>\frac{\delta}{2}+\frac{f\left(x+t_{i_{k_{j}}} v_{i_{k_{j}}}, y_{0}+t_{i_{k_{j}}} w\right)-f\left(x, y_{0}+t_{i_{k_{j}}} w\right)}{t_{i_{k_{j}}}} \quad$ (by definition of limit),

(2) $0<t_{i_{k_{j}}}<\varepsilon$

(3) $0<t_{i_{k_{j}}}<\min \left(\frac{\eta_{0}}{4} ; \frac{\eta_{0}}{2 M}\right)$

(possible since $t_{i_{k_{j}}} \downarrow 0$ ),

(4) $d_{M}\left(v_{i_{k_{j}}}, v\right)<\varepsilon$

(idem),

(possible since $v_{i_{k_{j}}} \stackrel{j}{\rightarrow} v$ and $\left.v_{i_{k_{j}}} \in \bar{B}(0 ; M)\right)$.

Relabel $\left\{t_{i_{k_{j}}}\right\}_{j \geq j_{0}} \equiv\left\{t_{j}\right\}_{j=1}^{\infty}$ and $\left\{v_{i_{k_{j}}}\right\}_{j \geq j_{0}} \equiv\left\{v_{j}\right\}_{j=1}^{\infty}$. And let us look at all points $y$ in $C \cap B\left(y_{0} ; \frac{\eta_{0}}{2}\right)$. In particular, we have: $\frac{f\left(x+t v^{\prime}, y\right)-f(x, y)}{t}>r$ if $0<t<\varepsilon$ and if $d_{M}\left(v^{\prime}, v\right)<\varepsilon$. Hence, by (2) and (4), for every $j=1,2, \ldots$, we have:

$$
\frac{f\left(x+t_{j} v_{j}, y\right)-f(x, y)}{t_{j}}>r \text {. }
$$

Combining with (1), we obtain after simplification, for each $j=1,2, \ldots$,

$$
\frac{f\left(x+t_{j} v_{j}, y\right)-f\left(x+t_{j} v_{j}, y_{0}+t_{j} w\right)}{t_{j}}+\frac{f\left(x, y_{0}+t_{j} w\right)-f(x, y)}{t_{j}}>\frac{\delta}{2} .
$$

Now since each of the preceding arguments of $f$ are points in $\left(x, y_{0}\right)+\eta_{0} B$ [by condition (3) and because $\left\|y-y_{0}\right\|<\frac{\eta_{0}}{2}$ and $\left.\|w\|=1\right]$, we get:

$$
\begin{gathered}
\frac{\delta}{2}<K_{0} \frac{\left\|y-\left(y_{0}+t_{j} w\right)\right\|}{t_{j}}+K_{0} \frac{\left\|y-\left(y_{0}+t_{j} w\right)\right\|}{t_{j}}, \forall j=1,2, \ldots, \\
\text { i.e. } \quad\left\|y-\left(y_{0}+t_{j} w\right)\right\|>\frac{\delta}{4 K_{0}} t_{j}, \quad \forall j=1,2, \ldots
\end{gathered}
$$

The end of the proof is identical to that of Part A of Theorem 3.1. 
Part B. To prove now that the set where $(\star \star)$ is satisfied is staunch in $U \times V_{n}$, denote: $S:=\left\{(x, y) \in U \times V_{n}:(\star \star)\right.$ is false $\}$ and

$$
C(v, w, r, M, \varepsilon):=\left\{(x, y) \in S:(\bullet) \text { is satisfied for all } t \text { in }(0, \varepsilon) \text { and } d_{M}\left(v^{\prime}, v\right)<\varepsilon\right\}
$$

where $(\bullet)$ is the following:

$$
\begin{aligned}
& \frac{f\left(x+t v^{\prime}, y\right)-f(x, y)}{t}>r>\inf _{\substack{\left\{v_{i}\right\} \stackrel{i}{\rightarrow} v \\
v_{i} \in \bar{B}(0 ; M) \\
\left\{t_{i}\right\} \downarrow 0}} \liminf _{i \rightarrow+\infty} \frac{f\left(x+t_{i} v_{i}, y+t_{i} w\right)-f(x, y)}{t_{i}} \ldots \\
& \ldots-\underline{D} f(x, y ; 0, w) \text {. }
\end{aligned}
$$

Then we easily show, as before, that $S=\bigcup_{(v, w, r, M, \varepsilon) \in D \times D^{\prime} \times \mathbb{Q} \times \mathbb{N} \times \mathbb{Q}_{+}^{*}} C(v, w, r, M, \varepsilon)$.

It remains to show that each $C \equiv C(v, w, r, M, \varepsilon)$ is porous. Let $\left(x_{0}, y_{0}\right)$ be a point in $C$, and denote by $K_{0}>0$ a Lipschitz constant of $f$ on $\left(x_{0}, y_{0}\right)+\eta_{0} B \subseteq$ $U \times V_{n}$, where $\eta_{0}>0$ and $B$ is the open unit ball in $H \times \mathbb{R}^{n}$. As before, we can find a sequence $\left\{v_{j}\right\}$ in $\left(\bar{B}(0 ; M), d_{M}\right)$ converging to $v$ and a real positive sequence $\left\{t_{j}\right\}$ decreasing to 0 such that for all $j=1,2, \ldots$ :

(1) $r>\frac{\delta}{2}+\frac{f\left(x_{0}+t_{j} v_{j}, y_{0}+t_{j} w\right)-f\left(x_{0}, y_{0}+t_{j} w\right)}{t_{j}}$,

(2) $0<t_{j}<\varepsilon$

(3) $0<t_{j}<\min \left(\frac{\eta_{0}}{8} ; \frac{\eta_{0}}{4 M}\right)$,

(4) $d_{M}\left(v_{j}, v\right)<\varepsilon$.

Now let us look at all points $(x, y)$ in $C \cap B\left(\left(x_{0}, y_{0}\right) ; \frac{\eta_{0}}{4}\right)$. We therefore have by (2) and (4), for each $j=1,2, \ldots$ :

$$
\frac{f\left(x+t_{j} v_{j}, y\right)-f(x, y)}{t_{j}}>r .
$$

Combining with (1), we obtain after simplification, for each $j=1,2, \ldots$,

$$
\frac{f\left(x+t_{j} v_{j}, y\right)-f\left(x_{0}+t_{j} v_{j}, y_{0}+t_{j} w\right)}{t_{j}}+\frac{f\left(x_{0}, y_{0}+t_{j} w\right)-f(x, y)}{t_{j}}>\frac{\delta}{2} \text {. }
$$

Now since each of the preceding arguments of $f$ are points in $\left(x_{0}, y_{0}\right)+\eta_{0} B$ [by condition (3) and because $\left\|(x, y)-\left(x_{0}, y_{0}\right)\right\|_{H \times \mathbb{R}^{n}}<\frac{\eta_{0}}{4}$ and $\|w\|=1$ ], we get for all $j=1,2, \ldots$ :

$$
\begin{gathered}
\frac{\delta}{2}<K_{0} \frac{\sqrt{\left\|x-x_{0}\right\|_{H}^{2}+\left\|y-\left(y_{0}+t_{j} w\right)\right\|_{\mathbb{R}^{n}}^{2}}}{t_{j}}+K_{0} \frac{\sqrt{\left\|x-x_{0}\right\|_{H}^{2}+\left\|y-\left(y_{0}+t_{j} w\right)\right\|_{\mathbb{R}^{n}}^{2}}}{t_{j}} \\
\text { i.e. } \quad\left\|(x, y)-\left(x_{0}, y_{0}+t_{j} w\right)\right\|_{H \times \mathbb{R}^{n}}>\frac{\delta}{4 K_{0}} t_{j}, \quad \forall j=1,2, \ldots .
\end{gathered}
$$

The end of the proof is identical to that of Part B of Theorem 3.1.

Let us now rewrite Theorem 3.2 in a form which explicitly refers to nonsmooth partial subdifferentiation.

Theorem 3.3. Let $H$ be a real separable nontrivial Hilbert space and let $f: H \times \mathbb{R}^{n} \longrightarrow \mathbb{R}$ (with $n \geq 1$ ) be locally Lipschitz.

Then there exists a staunch Borel subset $S$ of $H \times \mathbb{R}^{n}$ such that for any $x$ in $H$, the set $\left\{y \in \mathbb{R}^{n}:(x, y) \in S\right\}$ is staunch in $\mathbb{R}^{n}$ and such that for each $(x, y)$ in $S$, we have:

$$
\partial_{D} f(x, y)=\partial_{D / x} f(x, y) \times \partial_{D / y} f(x, y)
$$


Proof. One inclusion is obvious. For the opposite one, suppose that $(\xi, \zeta)$ belongs to $\partial_{D / x} f(x, y) \times \partial_{D / y} f(x, y)$ with $(x, y)$ in the set $S$ given by Theorem 3.2. Then for any $(v, w)$ in $H \times \mathbb{R}^{n}$, we have:

$$
\begin{aligned}
f_{D}^{\prime}(x, y ; v, w) & \geq f_{D}^{\prime}(x, y ; v, 0)+\underline{D} f(x, y ; 0, w) \\
& =(f(\cdot, y))_{D}^{\prime}(x ; v)+\underline{D}(f(x, \cdot))(y ; w) \\
& \geq\langle\xi, v\rangle_{H}+\langle\zeta, w\rangle_{\mathbb{R}^{n}} .
\end{aligned}
$$

Thus $(\xi, \zeta)$ is in $\partial_{D} f(x, y)$.

\section{Differentiation Results in $\mathbb{R}^{n}$}

Let us recall that for locally Lipschitz functions on $\mathbb{R}^{n}$ (with $n \geq 1$ ), there is no distinction between Gâteaux and Fréchet differentiability and between the lower classical Dini derivate and the weak Dini derivate. Moreover, a staunch set is in particular a residual set of full $\lambda_{n}$-measure.

Theorem 4.1. Let $f: \mathbb{R}^{n} \longrightarrow \mathbb{R}$ be a locally Lipschitz function.

Then staunchly in $x$, the function $\underline{D} f(x ; \cdot)$ is concave in the direction.

Proof. Consider the following function defined on the whole of $\mathbb{R}^{n} \times \mathbb{R}^{n}$ :

$$
\begin{aligned}
h: & \mathbb{R}^{n} \times \mathbb{R}^{n} \longrightarrow \mathbb{R}, \\
& (x, t) \longmapsto h(x, t):=f(x+t) .
\end{aligned}
$$

By Theorem 3.1, $h$ being locally Lipschitz on $\mathbb{R}^{n} \times \mathbb{R}^{n}$, for $x=0$, the set

$$
\left\{t \in \mathbb{R}^{n}: \underline{D} h(0, t ; \alpha, \beta) \geq \underline{D} h(0, t ; \alpha, 0)+\underline{D} h(0, t ; 0, \beta) \forall(\alpha, \beta) \in \mathbb{R}^{n} \times \mathbb{R}^{n}\right\}
$$

is staunch in $\mathbb{R}^{n}$. But for all $(\alpha, \beta)$ in $\mathbb{R}^{n} \times \mathbb{R}^{n}$, we have:

$$
\underline{D} h(0, t ; \alpha, \beta)=\underline{D} f(t ; \alpha+\beta) \text {. }
$$

Hence, the following set is staunch in $\mathbb{R}^{n}$ :

$$
\left\{t \in \mathbb{R}^{n}: \underline{D} f(t ; u+w) \geq \underline{D} f(t ; u)+\underline{D} f(t ; w) \quad \forall(u, w) \in \mathbb{R}^{n} \times \mathbb{R}^{n}\right\} .
$$

By positive homogeneity of $\underline{D} f$ in the direction, we get the result.

Corollary 4.2. Let $f: \mathbb{R}^{n} \longrightarrow \mathbb{R}$ be a locally Lipschitz function such that $\underline{D} f(x ; \cdot)=\bar{D} f(x ; \cdot)$ (in other words, the one-sided directional derivate of $f$ at $x$ exists) for all $x$ in respectively a staunch set, a residual set, a set of full $\lambda_{n}$-measure. Then $f$ is differentiable on respectively a staunch set, a residual set, a set of full $\lambda_{n}$-measure.

Proof. By Theorem 4.1 applied to the locally Lipschitz functions $f$ and $(-f)$, we have: for all $x$ in the intersection of a staunch set with respectively a staunch set, a residual set, a set of full $\lambda_{n}$-measure, the function $\underline{D} f(x ; \cdot)=\bar{D} f(x ; \cdot)$ is concave and convex, hence linear, implying that $f^{\prime}(x)$ exists in respectively a staunch set, a residual set, a set of full $\lambda_{n}$-measure.

Let us apply this last corollary to three useful classes of functions (Definitions 4.1, 4.2 and 4.3 below):

Definition 4.1. Let $J=\{1,2, \ldots, n\}$. Let $f_{j}$ be $C^{1}$ functions on $\mathbb{R}^{n}$ (hence locally Lipschitz) for $j=1,2, \ldots, n$, Then the function $f$ defined by $f(x):=\max _{j \in J} f_{j}(x)$ for all $x$ in $\mathbb{R}^{n}$ is called a regular upper envelope of a finite family.

We recall: 
Proposition 4.3 (Danskin's Theorem [8]). Let $f$ be a regular upper envelope of a finite family on $\mathbb{R}^{n}$. Then $f$ is a locally Lipschitz function and for all $(x, v)$ in $\mathbb{R}^{n} \times \mathbb{R}^{n}$, we have:

$$
\underline{D} f(x ; v)=\bar{D} f(x ; v)=\max \left\{\left\langle f_{j}^{\prime}(x), v\right\rangle: j \in J(x)\right\},
$$

where $J(x)=\left\{j \in J: \max _{j \in J} f_{j}(x)=f_{j}(x)\right\}$.

A simple application of Corollary 4.2 and Proposition 4.3 gives:

Corollary 4.4. Let $f$ be a regular upper envelope of a finite family on $\mathbb{R}^{n}$. Then $f$ is differentiable on a staunch set of points.

The most prized functions in optimization are convex ones. Let us recall:

Definition 4.2. A function $f$ on $\mathbb{R}^{n}$ is said to be convex if for any $\lambda$ in $[0,1]$ and for any $(x, y)$ in $\mathbb{R}^{n} \times \mathbb{R}^{n}$, we have:

$$
f(\lambda x+(1-\lambda) y) \leq \lambda f(x)+(1-\lambda) f(y) .
$$

Proposition 4.5 (see [7] and [19]). Let $f$ be a convex function on $\mathbb{R}^{n}$. Then $f$ is locally Lipschitz and for all $(x, v)$ in $\mathbb{R}^{n} \times \mathbb{R}^{n}$, we have:

$$
\underline{D} f(x ; v)=\bar{D} f(x ; v)=\inf _{t>0} \frac{f(x+t v)-f(x)}{t} .
$$

A simple application of Corollary 4.2 and Proposition 4.5 now gives:

Corollary 4.6. Let $f: \mathbb{R}^{n} \longrightarrow \mathbb{R}$ be a convex function.

Then $f$ is differentiable on a staunch set of points.

Remark 4.1. For $n=1$, it is known that convex functions are differentiable everywhere except on a countable set of points, hence on a staunch set. But the result appears new for $n \geq 2$.

Definition 4.3. Let $F$ be a subset of $\mathbb{R}^{n}$. The function $d_{F}$ defined by $d_{F}(x):=$ $\inf _{y \in F}\|x-y\|$ for all $x$ in $\mathbb{R}^{n}$ is called the distance function associated to the set $F$.

Proposition 4.7 (see [7], [8], [17] and [28]). Let $F$ be a subset of $\mathbb{R}^{n}$. Then:

(a) $d_{F}$ is globally Lipschitz with Lipschitz constant 1 on $\mathbb{R}^{n}$.

(b) $d_{F}$ is convex if and only if $F$ is convex.

(c) If $F$ is closed, then for all $x \notin F$, we have:

$$
\underline{D} d_{F}(x ; v)=\bar{D} d_{F}(x ; v) \quad \forall v \in \mathbb{R}^{n} .
$$

A simple application of Corollary 4.2 and Proposition 4.7 leads to:

Corollary 4.8. Let $F$ be a closed (but not necessarily convex) set in $\mathbb{R}^{n}$. Then $d_{F}$ is staunchly differentiable in the open set $\mathbb{R}^{n} \backslash F$.

Proof. By Theorem 4.1, the function $d_{F}$ being Lipschitz, there exists a staunch set $S$ on which $\underline{D} d_{F}$ and $\bar{D} d_{F}$ are respectively concave and convex in the direction. Hence, by Proposition 4.7, the set $S \cap\left(\mathbb{R}^{n} \backslash F\right)$ is a staunch set in $\mathbb{R}^{n} \backslash F$ on which $\underline{D} d_{F}=\bar{D} d_{F}$ is linear in the direction. Thus staunchly in $\mathbb{R}^{n} \backslash F$, we obtain the desired differentiability of $d_{F}$. 
Remark 4.2. In [6], Clarke showed that for a closed set $F$ in $\mathbb{R}^{n}$, if $d_{F}$ is differentiable at $x \in H$, then the set

$$
\operatorname{proj}_{F}(x):=\left\{y \in F: d_{F}(x)=\|y-x\|\right\}
$$

is a singleton. So by Corollary 4.8 , we get unique projection on $F$ staunchly.

Another notion of differentiability, besides those two defined in Section 1, is that of intermediate subdifferentiability [11].

Definition 4.4. We say that a function $f: \mathbb{R}^{n} \longrightarrow \mathbb{R}$ is intermediately subdifferentiable at $x$ if there exists $\xi_{x}$ in $\mathbb{R}^{n}$ such that

$$
\underline{D} f(x ; v) \leq\left\langle\xi_{x}, v\right\rangle \leq \bar{D} f(x ; v) \quad \forall v \in \mathbb{R}^{n} .
$$

Theorem 4.9. Let $f: \mathbb{R}^{n} \longrightarrow \mathbb{R}$ be a locally Lipschitz function. Then on a staunch set of points, $f$ is intermediately subdifferentiable.

Remark 4.3. Fabián and Preiss [11] proved in particular intermediate subdifferentiability of $f$ for all $x$ in a residual set of $\mathbb{R}^{n}$, but Theorem 4.9 appears new and implies the latter.

Proof. Let $S$ be the staunch set where $\underline{D} f$ and $\bar{D} f$ are respectively concave and convex in the direction (by Theorem 4.1 applied to $f$ and $(-f)$ ). Let $x$ be given in $S$. Define $g(v)=\underline{D} f(x ; v)$ and $h(v)=\bar{D} f(x ; v)$ for all $v$ in $\mathbb{R}^{n}$. Of course, $g$ and $h$ are Lipschitz with constant $K_{x}>0$ (by property (d) of Proposition 2.2 of the classical Dini derivates) and satisfy $g \leq h$. Suppose there exists $v_{0}$ in $\mathbb{R}^{n}$ such that $g\left(v_{0}\right)<h\left(v_{0}\right)$ (otherwise, $g=h$ is linear and the result is obvious). Consider the convex sets $A:=\left\{(v, r) \in \mathbb{R}^{n} \times \mathbb{R}: r>h(v)\right\}$ and $B:=$ hypo $g=\left\{(v, r) \in \mathbb{R}^{n} \times \mathbb{R}\right.$ : $r \leq g(v)\}$. Since $A$ is open and $A \cap B=\emptyset$, we can separate these two nonempty convex sets [8], i.e. we can find $\left(\xi_{1}, \xi_{2}\right)$ in $\left(\mathbb{R}^{n} \times \mathbb{R}\right) \backslash\{(0,0)\}$ and $\alpha$ in $\mathbb{R}$ such that:

$$
\begin{gathered}
\left\langle\left(\xi_{1}, \xi_{2}\right),(v, r)\right\rangle<\alpha \quad \forall(v, r) \in A \\
\text { and }\left\langle\left(\xi_{1}, \xi_{2}\right),(v, r)\right\rangle \geq \alpha \quad \forall(v, r) \in B .
\end{gathered}
$$

Denoting by epi $h$ the closure of $A$, we get:

$$
\begin{gathered}
\left\langle\left(\xi_{1}, \xi_{2}\right),(v, r)\right\rangle \leq \alpha \quad \forall(v, r) \in \text { epi } h \\
\text { and }\left\langle\left(\xi_{1}, \xi_{2}\right),(v, r)\right\rangle \geq \alpha \quad \forall(v, r) \in \text { hypo } g .
\end{gathered}
$$

Since $(0,0)$ belongs to epi $h \cap$ hypo $g$, we deduce that $\alpha=0$, implying that $\xi_{2} \neq 0$. Now since $g\left(v_{0}\right)<h\left(v_{0}\right)$, we easily deduce that $\xi_{2}<0$. Setting $\xi=\xi_{1} /\left(-\xi_{2}\right)$, we obtain:

$$
\langle\xi, v\rangle \leq h(v) \quad \text { and } \quad\langle\xi, v\rangle \geq g(v) \quad \forall v \in \mathbb{R}^{n},
$$

i.e. $f$ is intermediately subdifferentiable at $x$.

Let us now prove some nonsmooth partial subdifferentiation formulas:

Theorem 4.10. Let $f: \mathbb{R}^{n} \longrightarrow \mathbb{R}$ be a locally Lipschitz function. Then staunchly in $x=\left(x_{1}, \ldots, x_{n}\right)$, we have:

$$
\partial_{D} f(x)=\prod_{i=1}^{n} \partial_{D / x_{i}} f(x) .
$$


Proof. Of course, $\partial_{D} f(x)$ is always contained in $\prod_{i=1}^{n} \partial_{D / x_{i}} f(x)$. For the opposite inclusion, let $x=\left(x_{1}, \ldots, x_{n}\right)$ belong to the staunch set on which $\underline{D} f$ is suradditive in the direction (see Theorem 4.1), let $\xi=\left(\xi_{1}, \ldots, \xi_{n}\right)$ belong to $\prod_{i=1}^{n} \partial_{D / x_{i}} f(x)$ and let $v=\left(v_{1}, \ldots, v_{n}\right)$ be an arbitrary vector in $\mathbb{R}^{n}$. We have, by definition of partial D-subdifferentials, for $i=1, \ldots, n$ :

$\xi_{i} v_{i} \leq \underline{D} f\left(x_{1}, \ldots, x_{i-1}, \bullet, x_{i+1}, \ldots, x_{n}\right)\left(x_{i} ; v_{i}\right)=\underline{D} f\left(x ;\left(0, \ldots, 0, v_{i}, 0, \ldots, 0\right)\right)$.

Summing up for $i=1, \ldots, n$ and using suradditivity of $\underline{D} f(x ; \cdot)$, we obtain:

$$
\langle\xi, v\rangle=\sum_{i=1}^{n} \xi_{i} v_{i} \leq \underline{D} f\left(x ; \sum_{i=1}^{n}\left(0, \ldots, 0, v_{i}, 0, \ldots, 0\right)\right)=\underline{D} f(x ; v) .
$$

This implies that $\xi$ belongs to $\partial_{D} f(x)$.

If we are given a basis, say $\left\{e_{1}, \ldots, e_{n}\right\}$, of $\mathbb{R}^{n}$, then we define the gradient of $f$ at $x$ to be the vector in $\mathbb{R}^{n}$ :

$$
\nabla_{n} f(x):=\left(\frac{\partial f}{\partial x_{1}}(x), \ldots, \frac{\partial f}{\partial x_{n}}(x)\right), \text { whenever it exists. }
$$

Corollary 4.11. Let $f: \mathbb{R}^{n} \longrightarrow \mathbb{R}$ be locally Lipschitz. If $\nabla_{n} f(x)$ exists for all $x$ in respectively a staunch set, a residual set, a set of full $\lambda_{n}$-measure, then $f^{\prime}(x)$ exists for all $x$ in respectively a staunch set, a residual set, a set of full $\lambda_{n}$-measure.

Proof. For each $x=\left(x_{1}, \ldots, x_{n}\right)$ in the staunch set given by Theorem 4.10 , we have

$$
\partial_{D} f(x)=\prod_{i=1}^{n} \partial_{D} / x_{i} f(x) .
$$

But by Proposition 2.1, for each $i=1, \ldots, n$, we have:

$$
\partial_{D} / x_{i} f(x)=\left\{\frac{\partial f}{\partial x_{i}}(x)\right\}
$$

Now since a staunch subset of $\mathbb{R}^{n}$ is in particular a residual set of full $\lambda_{n}$-measure (see Section 2), we deduce that the D-subdifferential $\partial_{D} f(x)$ is equal to the singleton $\left\{\nabla_{n} f(x)\right\}$ for all $x$ in respectively a staunch set, a residual set, a set of full $\lambda_{n^{-}}$ measure. Applying the same reasoning to the locally Lipschitz function $(-f)$ and remembering Proposition 2.1, we obtain the result.

The next corollary is the celebrated theorem of Rademacher:

Corollary 4.12 (Rademacher's Theorem (see [18], [23] and [24]). Let $f: \mathbb{R}^{n} \longrightarrow$ $\mathbb{R}$ be locally Lipschitz. Then $f$ is differentiable on a set of full $\lambda_{n}$-measure.

Proof. For $n=1$, we know that $\frac{\partial f}{\partial x}(x)=\nabla_{1} f(x)=f^{\prime}(x)$ exists for $\lambda_{1}$-almost all $x$ in $\mathbb{R}$. For $n \geq 2$, a simple Fubini argument on iterated integration gives the existence of the gradient outside a $\lambda_{n}$-null set in $\mathbb{R}^{n}$. Of course, the mere existence of $\nabla_{n} f(x)$ does not imply that it is equal to $f^{\prime}(x)$, nor that it belongs to $\partial_{D} f(x)$, or even that the latter is nonempty. However, using Corollary 4.11, we easily get the result.

From Theorem 4.10, we derive Corollaries 4.11 and 4.12. However, for a locally Lipschitz function $f$ on $\mathbb{R}^{2}$, we could use the stronger nonsmooth partial subdifferentiation formula given by Theorem 3.3, namely: there exists a staunch set $S$ of 
$\mathbb{R}^{2}$ such that for any $x$ in $\mathbb{R}$, the set $\{y \in \mathbb{R}:(x, y) \in S\}$ is staunch in $\mathbb{R}$, and such that for any $(x, y)$ in $S$, we have

$$
\partial_{D} f(x, y)=\partial_{D / x} f(x, y) \times \partial_{D / y} f(x, y) .
$$

Exploiting this, we can prove for example the following:

Corollary 4.13. Let $f$ be a locally Lipschitz function on $\mathbb{R}^{2}$ such that $\frac{\partial f}{\partial x} f(x, y)$ exists for all $(x, y)$ in $\mathbb{R}^{2}$. Then for all $x$ in $\mathbb{R}$, the set $\left\{y \in \mathbb{R}: f^{\prime}(x, y)\right.$ exists $\}$ is of full $\lambda_{1}$-measure.

(The hypothesis and the conclusion are both stronger than that of Rademacher's Theorem.)

Corollary 4.14. Let $f$ be a locally Lipschitz function on $\mathbb{R}^{2}$ such that for all $x$ in $\mathbb{R}$, the set $\{y \in \mathbb{R}: \nabla f(x, y)$ exists $\}$ is a residual set of $\mathbb{R}$; and let $G$ be any residual set of $\mathbb{R}^{2}$. Then the set $\left\{(x, y) \in G: f^{\prime}(x, y)\right.$ exists $\}$ is dense in $\mathbb{R}^{2}$.

Proof. By the Kuratowski-Ulam Theorem [14], there exists a residual set $G_{1}$ in $\mathbb{R}$ such that for all $x$ in $G_{1}$, the set $\{y \in \mathbb{R}:(x, y) \in G\}$ is a residual set in $\mathbb{R}$. Using Theorem 3.3, it is easy to prove the density of the intersection of $G$ with the set where the derivative of $f$ exists.

We recall the classical result that if $f$ is a function on $\mathbb{R}^{n}$, if all its partial derivatives exist and all but one are continuous at the point $x_{0}=\left(x_{1}^{0}, \ldots, x_{n}^{0}\right)$, then the derivative at the point $x_{0}$ exists and we have the smooth partial differentiation formula:

$$
f^{\prime}\left(x_{0}\right)=\left(\frac{\partial f}{\partial x_{1}}\left(x_{0}\right), \ldots, \frac{\partial f}{\partial x_{n}}\left(x_{0}\right)\right) .
$$

When $f$ is locally Lipschitz, we now see that this result is a special case of the following new fact in nonsmooth analysis:

Theorem 4.15. Let $f: \mathbb{R}^{n}, n \geq 2 \longrightarrow \mathbb{R}$ be locally Lipschitz, let $x_{0}=\left(x_{1}^{0}, \ldots, x_{n}^{0}\right)$ be a point in $\mathbb{R}^{n}$ and let the function $y \longmapsto \underline{D} f\left(x_{1}^{0}, \ldots, x_{i-1}^{0}, y, x_{i+1}^{0}, \ldots, x_{n}^{0} ; v\right)$ be continuous at $x_{i}^{0}$, for $i=1,2, \ldots, n-1$ and for all $v$ in $\mathbb{R}^{n}$. Then we have:

$$
\partial_{D} f\left(x_{0}\right)=\prod_{i=1}^{n} \partial_{D / x_{i}} f\left(x_{0}\right) .
$$

In particular, if all partial D-subdifferentials are not empty at the point $x_{0}$, then $\partial_{D} f\left(x_{0}\right)$ is not empty.

Proof. Let $v=\left(v_{1}, \ldots, v_{n}\right)$ be given in $\mathbb{R}^{n}$. By Theorem 3.1, the set

$$
\begin{aligned}
S_{1}:=\left\{y \in \mathbb{R}: \underline{D} f\left(y, x_{2}^{0}, \ldots, x_{n}^{0} ; v_{1}, v_{2}, \ldots, v_{n}\right)\right. & \\
\geq \underline{D} f\left(y, x_{2}^{0}, \ldots, x_{n}^{0}\right. & \left.; v_{1}, 0, \ldots, 0\right) \\
& \left.+\underline{D} f\left(y, x_{2}^{0}, \ldots, x_{n}^{0} ; 0, v_{2}, \ldots, v_{n}\right)\right\}
\end{aligned}
$$

is staunch in $\mathbb{R}$. Hence taking a sequence $\left\{y_{k}\right\} \subseteq S_{1}$ converging to $x_{1}^{0}$ and using the continuity hypothesis on $\underline{D} f$, we have:

$$
\begin{array}{rl}
\underline{D} f\left(x_{1}^{0}, x_{2}^{0}, \ldots, x_{n}^{0} ; v_{1}, v_{2}, \ldots, v_{n}\right) \geq \underline{D} & f\left(x_{1}^{0}, x_{2}^{0}, \ldots, x_{n}^{0} ; v_{1}, 0, \ldots, 0\right) \\
& +\underline{D} f\left(x_{1}^{0}, x_{2}^{0}, \ldots, x_{n}^{0} ; 0, v_{2}, \ldots, v_{n}\right) .
\end{array}
$$


Using the same reasoning with the staunch sets

$$
\begin{aligned}
S_{j}:=\{y \in \mathbb{R}: & \underline{D} f\left(x_{1}^{0}, x_{2}^{0}, \ldots, x_{j-1}^{0}, y, x_{j+1}^{0}, \ldots, x_{n}^{0} ; 0, \ldots, 0, v_{j}, \ldots, v_{n}\right) \\
\geq & \underline{D} f\left(x_{1}^{0}, x_{2}^{0}, \ldots, x_{j-1}^{0}, y, x_{j+1}^{0}, \ldots, x_{n}^{0} ; 0, \ldots, 0, v_{j}, 0, \ldots, 0\right) \\
& \left.+\underline{D} f\left(x_{1}^{0}, x_{2}^{0}, \ldots, x_{j-1}^{0}, y, x_{j+1}^{0}, \ldots, x_{n}^{0} ; 0, \ldots, 0,0, v_{j+1}, \ldots, v_{n}\right)\right\},
\end{aligned}
$$

for $j=2, \ldots, n-1$, we get:

$$
\underline{D} f\left(x_{0} ; v\right) \geq \sum_{i=1}^{n} \underline{D} f\left(x_{0} ;\left(0, \ldots, 0, v_{i}, 0, \ldots, 0\right)\right) .
$$

Following the proof of Theorem 4.10, we obtain the result.

\section{Gâteaux Differentiability}

Let us suppose $H$ is separable with countable orthonormal basis $\left\{e_{1}, e_{2}, \ldots\right\}$. We shall identify $H$ with the space $\left\{\left(x_{1}, x_{2}, \ldots\right): \sum_{i=1}^{\infty}\left|x_{i}\right|^{2}<\infty\right\}$. We now refer to $\nabla_{n} f(x)$ as the vector in $H$ whose $n$ first "coordinates" are the $n$ first partial derivatives of $f$ at $x$, but whose subsequent coordinates are zero, i.e.:

$$
\nabla_{n} f(x)=\left(\frac{\partial f}{\partial x_{1}}(x), \ldots, \frac{\partial f}{\partial x_{n}}(x), 0,0,0, \ldots\right), \text { whenever it exists. }
$$

Even if all partial derivatives of $f$ at $x$ exist, the vector $\left(\frac{\partial f}{\partial x_{1}}(x), \frac{\partial f}{\partial x_{2}}(x), \ldots\right)$ may not be an element of $H$, and of course might not equal $f_{G}^{\prime}(x)$ or $f_{F}^{\prime}(x)$. Still:

Theorem 5.1. Let $\left\{e_{1}, e_{2}, \ldots\right\}$ be a countable orthonormal basis of $H$. Let $f$ : $H \rightarrow \mathbb{R}$ be a locally Lipschitz function. Then $f_{G}^{\prime}(x)$ exists for all $x$ in a $\left\{e_{n}\right\}$-full set of $H$.

Theorem 5.1 is a well-known result [16]: it says in particular that a locally Lipschitz function on a separable Hilbert space is Gâteaux differentiable on a dense and uncountable set which is big in a measure theoretic sense (see Section 2). We will see that it is an easy consequence of Theorem 3.1.

Proof. • Let $n$ be given in $\mathbb{N}$. Denote $H_{n} \equiv\left\{\left(x_{n+1}, x_{n+2}, \ldots\right): \sum_{i=n+1}^{\infty}\left|x_{i}\right|^{2}<\infty\right\}$ (it is a real separable Hilbert space) and $(x, y) \equiv\left(\left(x_{n+1}, x_{n+2}, \ldots\right),\left(x_{1}, \ldots, x_{n}\right)\right)$ an element of $H \equiv H_{n} \times \mathbb{R}^{n}$. Consider the following two sets whose definitions explicitly refer to the basis $\left\{e_{n}\right\}$ :

$$
\begin{array}{r}
A_{n} \equiv\left\{(x, y) \in H_{n} \times \mathbb{R}^{n}: \underline{D} f(x, y ; v, w) \geq \underline{D} f(x, y ; v, 0)+\underline{D} f(x, y ; 0, w)\right. \\
\text { for all } \left.(v, w) \text { in } H_{n} \times \mathbb{R}^{n}\right\}
\end{array}
$$

and $A_{n}^{\prime} \equiv\left\{(x, y) \in H_{n} \times \mathbb{R}^{n}:\right.$ the function $y^{\prime} \longmapsto f\left(x, y^{\prime}\right)$

$$
\text { is Fréchet-differentiable at } \left.y^{\prime}=y\right\} \text {. }
$$

Let us show that $A_{n}$ and $A_{n}^{\prime}$ are $\left\{e_{n}\right\}$-full sets. It is easy to show that they are Borel sets (using Proposition 2.2 and [16]). Now let $a$ in $\mathbb{N}$ be given. With the same notations as in Section 2, the sets $A_{n} \cap K^{a}$ and $A_{n}^{\prime} \cap K^{a}$ are $\nu^{a}$-measurable; hence, for all $x$ in $\tilde{K}_{n}^{a}$, the sets $\left\{y \in K_{n}^{a}:(x, y) \in A_{n} \cap K^{a}\right\}$ and $\left\{y \in K_{n}^{a}:(x, y) \in A_{n}^{\prime} \cap K^{a}\right\}$ are $\mu_{n}^{a}$-measurable in $K_{n}^{a}$. We then have:

$$
\mu_{n}^{a}\left[\left\{y \in K_{n}^{a}:(x, y) \in A_{n}^{c} \cap K^{a}\right\}\right] \leq \lambda_{n}\left[\left\{y \in \mathbb{R}^{n}:(x, y) \notin A_{n}\right\}\right]=0,
$$


by Theorem 3.1 and Proposition 2.6. Also we have:

$$
\begin{aligned}
& \mu_{n}^{a}\left[\left\{y \in K_{n}^{a}:(x, y) \in A_{n}^{\prime c} \cap K^{a}\right\}\right] \leq \lambda_{n}\left[\left\{y \in \mathbb{R}^{n}:(x, y) \notin A_{n}^{\prime}\right\}\right] \\
& \quad=\lambda_{n}\left[\left\{y \in \mathbb{R}^{n}: y^{\prime} \longmapsto f\left(x, y^{\prime}\right) \text { is not Fréchet-differentiable at } y^{\prime}=y\right\}\right] \\
& \quad=0
\end{aligned}
$$

by Rademacher's Theorem (Corollary 4.12). Hence $\tilde{\mu}_{n}^{a} \otimes \mu_{n}^{a}\left(A_{n}^{c} \cap K^{a}\right)=\tilde{\mu}_{n}^{a} \otimes$ $\mu_{n}^{a}\left(A_{n}^{\prime c} \cap K^{a}\right)=0$, implying that $\nu^{a}\left(A_{n} \cap K^{a}\right)=\nu^{a}\left(A^{\prime}{ }_{n} \cap K^{a}\right)=1$. Thus $A_{n}$ and $A_{n}^{\prime}$ are $\left\{e_{n}\right\}$-full sets in $H_{n} \times \mathbb{R}^{n} \equiv H$.

- Let $A \equiv \bigcap_{n=1}^{\infty}\left(A_{n} \cap A_{n}^{\prime}\right)$. It is obviously a $\left\{e_{n}\right\}$-full set in $H$. Let us show that $A$ is the set we are looking for. Indeed, for given $x$ in $A$, we have:

- For each $n$ in $\mathbb{N}$ and for every $(v, w)$ in $H_{n} \times \mathbb{R}^{n}$, since $x$ is in $A_{n} \cap A_{n}^{\prime}$, we have in evident notation:

$$
\underline{D} f(x ; v, w)-\underline{D} f(x ; v, 0) \geq \underline{D} f(x ; 0, w)=\left\langle\nabla_{n} f(x),(v, w)\right\rangle .
$$

Hence using the $K_{x}$-Lipschitz condition of the classical Dini directional derivate, we get:

$$
\left\langle\nabla_{n} f(x),(v, w)\right\rangle \leq K_{x}\|(v, w)\|+K_{x}\|(v, 0)\| \leq 2 K_{x}\|(v, w)\| .
$$

The sequence $\left\{\nabla_{n} f(x)\right\}_{n=1}^{\infty}$ being weakly bounded in $H$, it is (strongly) bounded, using the Banach-Steinhaus Theorem. So taking a subsequence if necessary, we can find $\xi_{x}$ in $H$ such that $\nabla_{n} f(x) \stackrel{n}{\rightarrow} \xi_{x}$.

- For every $v=\left(v_{1}, v_{2}, \ldots\right)$ in $H$, and given $\varepsilon>0$, let us pick $n_{0}$ in $\mathbb{N}$ such that:

(a) $\left.\left\langle\nabla_{n_{0}} f(x), v\right\rangle\right\rangle\left\langle\xi_{x}, v\right\rangle-\frac{\varepsilon}{2}$ [by definition of weak limit],

(b) $\left\|\left(0, \ldots, 0, v_{n_{0}+1}, v_{n_{0}+2}, \ldots\right)\right\|<\frac{\varepsilon}{2 K_{x}}\left[\right.$ since $\left.\left(v_{1}, \ldots, v_{n}, 0,0, \ldots\right) \stackrel{n}{\rightarrow} v\right]$.

Since $x$ belongs to $A_{n_{0}} \cap A_{n_{0}}^{\prime}$, then:

$$
\begin{aligned}
\underline{D f}(x ; v) & \geq \underline{D f}\left(x ; 0, \ldots, 0, v_{n_{0}+1}, v_{n_{0}+2}, \ldots\right)+\left\langle\nabla_{n_{0}} f(x), v\right\rangle \\
& >-K_{x}\left\|\left(0, \ldots, 0, v_{n_{0}+1}, v_{n_{0}+2}, \ldots\right)\right\|+\left\langle\xi_{x}, v\right\rangle-\frac{\varepsilon}{2} \\
& >\left\langle\xi_{x}, v\right\rangle-\varepsilon .
\end{aligned}
$$

Letting $\varepsilon \downarrow 0$, we obtain an element $\xi_{x}$ in $H$ such that $\left\langle\xi_{x}, v\right\rangle \leq \underline{D} f(x ; v)$ for all $v$ in $H$.

- Applying the same reasoning to $(-f)$ which is locally Lipschitz, we get: for all $x$ in a $\left\{e_{n}\right\}$-full set $B$, there exists $\xi_{x}^{\prime}$ in $H$ such that

$$
\left\langle-\xi_{x}^{\prime}, v\right\rangle \geq \bar{D} f(x ; v) \quad \forall v \in H .
$$

Hence if $x$ is in $A \cap B$, then $\xi_{x}^{\prime}=-\xi_{x}$ and we obtain the theorem.

The results in Section 4 can easily be extended to the setting of real separable Hilbert spaces, provided we replace the word "differentiability" by "Gâteaux differentiability". We state, without proof, the following:

Theorem 5.2. Let $H$ be a real separable Hilbert space. Let $f: H \longrightarrow \mathbb{R}$ be a locally Lipschitz function. Then staunchly in $x$, the function $\underline{D} f(x ; \cdot)$ is concave in the direction.

Corollary 5.3. Let $H$ be a real separable Hilbert space. Let $f: H \longrightarrow \mathbb{R}$ be a locally Lipschitz function such that $\underline{D} f(x ; \cdot)=\bar{D} f(x ; \cdot)$ (in other words, $f$ admits a one-sided directional derivate at $x$ ) for all $x$ in respectively a staunch set, a residual set. Then the function $f$ is Gâteaux differentiable on respectively a staunch set, a residual set. 
We want to apply this last corollary to the three special classes of functions introduced in Section 4. Definitions 4.1 and 4.3 and Propositions 4.3 and 4.7 remain valid in a separable Hilbert space hence we obtain:

Corollary 5.4. Let $H$ be a real separable Hilbert space. Let $f: H \longrightarrow \mathbb{R}$ be a regular upper envelope of a finite family on $H$. Then $f$ is Gâteaux differentiable on a staunch set of points.

Corollary 5.5. Let $H$ be a real separable Hilbert space. Let $F$ be a nonempty closed set in $H$. Then $d_{F}$ is staunchly Gâteaux differentiable in the open set $H \backslash F$.

In the setting of Hilbert spaces (in which we are interested), Definition 4.2 is still valid, but Proposition 4.5 becomes:

Proposition 5.6 (see [7] and [19]). Let $H$ be a real Hilbert space and $f: H \longrightarrow \mathbb{R}$ be a convex function on $H$. Then the following properties are equivalent:

(a) $f$ is continuous.

(b) $f$ is locally Lipschitz.

(c) $f$ is locally bounded.

Also, we have $\underline{D} f(x ; \cdot)=\bar{D} f(x ; \cdot)$ for all $x$ in $H$.

For convex continuous functions on a separable Hilbert space with countable orthonormal basis $\left\{e_{1}, e_{2}, \ldots\right\}$, Theorem 5.1 implies their Gâteaux differentiability on a $\left\{e_{n}\right\}$-full set of points. It is well-known that convex continuous functions are Gâteaux differentiable on a residual set of $H$, but actually a stronger result holds. Namely, using Proposition 5.6 and Corollary 5.3, we have:

Corollary 5.7. Let $H$ be a real separable Hilbert space. Let $f: H \longrightarrow \mathbb{R}$ be a convex continuous function on $H$. Then $f$ is Gâteaux differentiable on a staunch set of points.

As for intermediate subdifferentiability, we can generalize the theorem of Fabián and Preiss [11] by replacing the word "residual set" by "staunch set":

Theorem 5.8. Let $H$ be a real separable Hilbert space. Let $f: H \longrightarrow \mathbb{R}$ be a locally Lipschitz function. Then on a staunch set of points, $f$ is intermediately subdifferentiable, i.e. staunchly in $x$, and there exists an element $\xi_{x}$ in $H$ such that

$$
\underline{D} f(x ; v) \leq\left\langle\xi_{x}, v\right\rangle \leq \bar{D} f(x ; v) \quad \forall v \in H .
$$

\section{Fréchet Differentiability}

We could think of a corresponding version of Theorem 5.1 dealing with Fréchet differentiability, using Theorem 3.2. But we must pay attention to the fact that even if $f: H \longrightarrow \mathbb{R}$ is Lipschitz, the weak Dini derivate is not always Lipschitz in the direction: it could be equal to $-\infty$. That's why we only have:

Theorem 6.1. Let $\left\{e_{1}, e_{2}, \ldots\right\}$ be a countable orthonormal basis of $H$. Let $f$ : $H \longrightarrow \mathbb{R}$ be locally Lipschitz. Then for all $x$ in a $\left\{e_{n}\right\}$-full set of $H, f_{G}^{\prime}(x)$ exists and we have:

(a) either $\partial_{D} f(x)=\left\{f_{G}^{\prime}(x)\right\} \quad$ [Best Case] or $f_{D}^{\prime}(x ; 0)=-\infty$;

(b) either $f_{F}^{\prime}(x)$ exists [Worst Case] or $(\epsilon f)_{D}^{\prime}(x ; 0)=-\infty$ with some $\epsilon$ in $\{-1,+1\}$. 
Remark 6.1. When $f: H \longrightarrow \mathbb{R}$ is locally Lipschitz, then " $f_{D}^{\prime}(x ; 0)=-\infty$ " is equivalent to " $f_{D}^{\prime}(x ; \cdot) \equiv-\infty$ ". It is interesting to note that $f_{D}^{\prime}(x ; 0)$ is always either 0 or $-\infty$, by positive homogeneity of the weak Dini derivate. When $H$ is infinite-dimensional, an example of $f_{D}^{\prime}(x ; 0)=-\infty$ is given by the Lipschitz function $f(x)=-\|x\|$ for all $x \in H$ (see [8]).

When $f: H \longrightarrow \mathbb{R}$ is lower semicontinuous, we already know that the D-subdifferential of $f$ is not empty on a dense set [9]. But on that set, the Gâteaux derivative may not belong to the D-subdifferential, or may not even exist. Assertion (a) of Theorem 6.1 gathers two extreme cases related to the structure of the D-subdifferential: either it is empty (the worst case being when the weak Dini derivate is not even finite) or it is a singleton (consisting of the Gâteaux derivative). Assertion (b) of Theorem 6.1 also gathers two extreme cases related to the existence of the Fréchet derivative, whenever the Gâteaux derivative exists: this is an alternative-type kind of result.

Theorem 6.1 does not assert Fréchet differentiability on a dense set, a celebrated result of David Preiss [21], but it may give added information on the set where the Fréchet derivative exists: we have linked Gâteaux differentiability and Fréchet differentiability by means of a new criterion, namely the finiteness of the weak Dini derivate.

Corollary 6.2. Let $\left\{e_{1}, e_{2}, \ldots\right\}$ be a countable orthonormal basis of $H$ and let $f: H \longrightarrow \mathbb{R}$ be locally Lipschitz such that $( \pm f)_{D}^{\prime}(x ; 0)=0$ for all $x$ in a $\left\{e_{n}\right\}$-full set of $H$ (for example outside a countable set).

Then $f_{F}^{\prime}(x)$ exists for all $x$ in a $\left\{e_{n}\right\}$-full set of $H$, hence a fortiori on a dense and uncountable set of $H$.

Proof of Theorem 6.1. The proof is quite similar to that of Theorem 5.1.

- Using the same notations as in Theorem 5.1, let $A$ be the $\left\{e_{n}\right\}$-full set where the Gâteaux derivative of $f$ exists. If $x$ is in $A$, then $\partial_{D} f(x) \subseteq\left\{f_{G}^{\prime}(x)\right\}$. For the opposite inclusion, for each $n$ in $\mathbb{N}$, denote:

$$
\begin{aligned}
& B_{n} \equiv\left\{(x, y) \in H_{n} \times \mathbb{R}^{n}: f_{D}^{\prime}(x, y ; v, w) \geq f_{D}^{\prime}(x, y ; v, 0)+\right. \underline{D} f(x, y ; 0, w) \\
&\left.\forall(v, w) \in H_{n} \times \mathbb{R}^{n}\right\} .
\end{aligned}
$$

By Theorem 3.2 and Proposition 2.6, reproducing the argument in Theorem 5.1, we can see that $B_{n}$ is a $\left\{e_{n}\right\}$-full set. Denote $B \equiv A \cap \bigcap_{n=1}^{\infty} B_{n}$; it is the $\left\{e_{n}\right\}$ full set we are looking for. Indeed, if $x$ is in $B$, then $f_{G}^{\prime}(x)$ exists. Moreover, if $f_{D}^{\prime}(x ; 0) \neq-\infty$, then $v \longmapsto f_{D}^{\prime}(x ; v)$ is a real-valued Lipschitz function with constant $K_{x}>0$. Therefore given $v=\left(v_{1}, v_{2}, \ldots\right)$ in $H$ and $\varepsilon>0$, let us pick $n_{0}$ in $\mathbb{N}$ such that

(a) $\left\langle\nabla_{n_{0}} f(x), v\right\rangle>\left\langle f_{G}^{\prime}(x), v\right\rangle-\frac{\varepsilon}{2}\left[\operatorname{since} \nabla_{n} f(x) \stackrel{n}{\rightarrow} f_{G}^{\prime}(x)\right]$,

(b) $\left\|\left(0, \ldots, 0, v_{n_{0}+1}, v_{n_{0}+2}, \ldots\right)\right\|<\frac{\varepsilon}{2 K_{x}} \quad\left[\right.$ since $\left.\left(v_{1}, \ldots, v_{n}, 0,0, \ldots\right) \stackrel{n}{\rightarrow} v\right]$.

Since $x$ belongs to $B_{n_{0}}$, in evident notation, we have:

$$
\begin{aligned}
f_{D}^{\prime}(x ; v) & \geq f_{D}^{\prime}\left(x ; 0, \ldots, 0, v_{n_{0}+1}, v_{n_{0}+2}, \ldots\right)+\underline{D} f\left(x ; v_{1}, \ldots, v_{n_{0}}, 0,0, \ldots\right) \\
& =f_{D}^{\prime}\left(x ; 0, \ldots, 0, v_{n_{0}+1}, v_{n_{0}+2}, \ldots\right)+\left\langle\nabla_{n_{0}} f(x), v\right\rangle \\
& \geq-K_{x}\left\|\left(0, \ldots, 0, v_{n_{0}+1}, v_{n_{0}+2}, \ldots\right)\right\|+\left\langle\nabla_{n_{0}} f(x), v\right\rangle \\
& >\left\langle f_{G}^{\prime}(x), v\right\rangle-\varepsilon
\end{aligned}
$$

Letting $\varepsilon \downarrow 0$, we get (a). 
- Applying the same reasoning to $(-f)$ which is locally Lipschitz, we get: for all $x$ in a $\left\{e_{n}\right\}$-full set, where $(-f)_{G}^{\prime}(x)=-f_{G}^{\prime}(x)$ exists,

$$
-f_{G}^{\prime}(x) \in \partial_{D}(-f)(x) \quad \text { or } \quad(-f)_{D}^{\prime}(x ; 0)=-\infty .
$$

Combining with (a), and remembering Proposition 2.1, we get (b).

Theorem 6.3. Let $H$ be a real separable Hilbert space. Let $f: H \longrightarrow \mathbb{R}$ be locally Lipschitz such that $f_{G}^{\prime}(x)$ exists staunchly in $x$. Then for all $x$ in a staunch set of $H$, we have:

(a) either $\partial_{D} f(x)=\left\{f_{G}^{\prime}(x)\right\}$ or $f_{D}^{\prime}(x ; 0)=-\infty$

[Best Case]

either $f_{F}^{\prime}(x)$ exists

or $(\epsilon f)_{D}^{\prime}(x ; 0)=-\infty$ with some $\epsilon$ in $\{-1,+1\}$.

Proof. The proof is quite similar to that of Theorem 6.1. Let $A$ be the staunch set where the Gâteaux derivative of $f$ exists. Using the same notations as in Theorem 6.1, for each $n$ in $\mathbb{N}$, denote:

$$
\begin{aligned}
B_{n} \equiv\left\{(x, y) \in H_{n} \times \mathbb{R}^{n}: f_{D}^{\prime}(x, y ; v, w) \geq f_{D}^{\prime}(x, y ; v, 0)+\underline{D} f(x, y ; 0, w)\right. \\
\left.\forall(v, w) \in H_{n} \times \mathbb{R}^{n}\right\} .
\end{aligned}
$$

By Theorem 3.2, $B_{n}$ is staunch in $H_{n} \times \mathbb{R}^{n} \equiv H$. Denote $B \equiv A \cap \bigcap_{n=1}^{\infty} B_{n}$; it is the staunch set we are looking for. Indeed, if $x$ is in $B$ and if $f_{D}^{\prime}(x ; 0) \neq-\infty$, then $v \longmapsto f_{D}^{\prime}(x ; v)$ is a real-valued Lipschitz function with constant $K_{x}>0$. Therefore given $v=\left(v_{1}, v_{2}, \ldots\right)$ in $H$ and $\varepsilon>0$, let us pick $n_{0}$ in $\mathbb{N}$ such that

(a) $\left\langle\nabla_{n_{0}} f(x), v\right\rangle>\left\langle f_{G}^{\prime}(x), v\right\rangle-\frac{\varepsilon}{2} \quad\left[\right.$ since $\left.\nabla_{n} f(x) \stackrel{n}{\rightarrow} f_{G}^{\prime}(x)\right]$,

(b) $\quad\left\|\left(0, \ldots, 0, v_{n_{0}+1}, v_{n_{0}+2}, \ldots\right)\right\|<\frac{\varepsilon}{2 K_{x}}\left[\right.$ since $\left.\left(v_{1}, \ldots, v_{n}, 0,0, \ldots\right) \stackrel{n}{\rightarrow} v\right]$.

Since $x$ belongs to $B_{n_{0}}$, in evident notation, we have:

$$
\begin{aligned}
f_{D}^{\prime}(x ; v) & \geq f_{D}^{\prime}\left(x ; 0, \ldots, 0, v_{n_{0}+1}, v_{n_{0}+2}, \ldots\right)+\underline{D} f\left(x ; v_{1}, \ldots, v_{n_{0}}, 0,0, \ldots\right) \\
& =f_{D}^{\prime}\left(x ; 0, \ldots, 0, v_{n_{0}+1}, v_{n_{0}+2}, \ldots\right)+\left\langle\nabla_{n_{0}} f(x), v\right\rangle \\
& \geq-K_{x}\left\|\left(0, \ldots, 0, v_{n_{0}+1}, v_{n_{0}+2}, \ldots\right)\right\|+\left\langle\nabla_{n_{0}} f(x), v\right\rangle \\
& >\left\langle f_{G}^{\prime}(x), v\right\rangle-\varepsilon
\end{aligned}
$$

Letting $\varepsilon \downarrow 0$, we get (a). The same reasoning applied to $(-f)$ gives (b).

Definition 6.1. With the same setting as in Theorem 6.1, we say that: $x$ satisfies property $(*)_{\mathbf{f}}$ if $f_{D}^{\prime}(x ; 0)=-\infty$, which amounts to saying that there exist a real positive sequence $\left\{t_{i}\right\}$ decreasing to 0 and a sequence $\left\{v_{i}\right\}$ of unit vectors converging weakly to 0 such that

$$
\lim _{i \rightarrow \infty} \frac{f\left(x+t_{i} v_{i}\right)-f(x)}{t_{i}}<0 .
$$

In the next section, we will consider special functions $f$ for which property $(*)_{f}$ is never satisfied. But for now, let us consider $(*)_{f}$. We will first give a geometric interpretation of property $(*)_{f}$. For that purpose, we define:

Definition 6.2. Let $x$ belong to a closed subset $S$ of a Hilbert space $H$. Then the weak Dini tangent cone of $S$ at $x$ is

$$
T_{S}^{D}(x):=\left\{v=\mathrm{w}-\lim _{i \rightarrow \infty} \frac{x_{i}-x}{t_{i}} \in H: x_{i} \in S \text { and } t_{i} \downarrow 0\right\} .
$$


Similarly, the weak Dini paratingent cone is

$$
P_{S}^{D}(x):=\left\{v=\mathrm{w}-\lim _{i \rightarrow \infty} \frac{y_{i}-x_{i}}{t_{i}} \in H:\left(x_{i}, y_{i}\right) \in S \times S, x_{i} \rightarrow x \text { and } t_{i} \downarrow 0\right\} .
$$

When $H=\mathbb{R}^{n}$, the weak Dini tangent cone is equal to the Bouligand contingent cone, and the weak Dini paratingent cone is equal to the Bouligand paratingent cone (see [5] and [26] for a definition of Bouligand cones); but when $H$ is infinite-dimensional, they may be different. When $H$ is separable, using Choquet's Theorem [5], Shi Shu Zhong has proved that on a residual set of $S$, the Bouligand contingent cone and the Bouligand paratingent cone are equal [26]. However, we do not know any result about the equality of the weak Dini tangent cone and the weak Dini paratingent cone, even though it is a simple application of Choquet's Theorem on abstract contingent and paratingent cones, as we now see:

Proposition 6.4. Let $S$ be a closed set of a real separable Hilbert space $H$. Then for all $x$ in a residual set of $S$, we have $T_{S}^{D}(x)=P_{S}^{D}(x)$.

Sketch of the proof (see [2]). Using exactly the notation of Choquet in [5] (Definition VII.1.5, p 181), we set $U:=(H,\|\cdot\|), P=E:=S$ and $\Delta:=\bar{B}(0 ; 1)$ the closed unit ball in $H$, which is metrizable with the weak topology (since $H$ is separable). Also for all $(x, y) \in P \times E$ such that $x \neq y$, set $\delta(x, y):=\{(y-x) /(\|y-x\|)\} \cup\{0\}$. Then Choquet's Theorem (VII.1.7, p 182 in [5]) implies that for all $x$ in a residual set of $P$, the following two sets are equal:

$$
\begin{gathered}
\mathcal{C}_{E}(x)=\left\{v=\mathrm{w}-\lim _{i \rightarrow+\infty} \frac{x_{i}-x}{\left\|x_{i}-x\right\|}: x_{i} \in S \backslash\{x\} \rightarrow x\right\} \cup\{0\} \text { and } \\
\mathcal{P}_{E, P}(x)=\left\{v=\mathrm{w}-\lim _{i \rightarrow+\infty} \frac{y_{i}-x_{i}}{\left\|y_{i}-x_{i}\right\|}:\left(x_{i}, y_{i}\right) \in(S \times S) \longrightarrow(x, x) \text { and } x_{i} \neq y_{i}\right\} \cup\{0\} .
\end{gathered}
$$

Hence $T_{S}^{D}(x)=\bigcup_{\lambda>0} \lambda \mathcal{C}_{E}(x)=\bigcup_{\lambda>0} \lambda \mathcal{P}_{E, P}(x)=P_{S}^{D}(x)$ for all $x$ in a residual set of $S$.

Remark 6.2. Since $P_{S}^{D}(x)$ is symmetric, Proposition 6.4 implies that $T_{S}^{D}(x)$ is also symmetric for all $x$ in a residual set of the closed set $S$. Moreover, passing to polars in Proposition 6.4, we obtain that the weak Dini normal cone [8] is generically a subspace of $H$.

We are now in a position to state:

Proposition 6.5. Let $H$ be a real separable Hilbert space, let $f: H \longrightarrow \mathbb{R}$ be a continuous function, and let $g r f:=\{(x, y) \in H \times \mathbb{R}: y=f(x)\}$ denote the graph of $f$. Then for all $x$ in a residual set of $H$, the following are equivalent:

(a) $x$ does not satisfy $(*)_{f}$.

(b) $(0,-1)$ does not belong to $T_{g r f}^{D}(x, f(x))$.

(c) $(0,+1)$ does not belong to $T_{g r f}^{D}(x, f(x))$.

(d) $(0,-1)$ does not belong to $T_{g r(-f)}^{D}(x,(-f)(x))$.

(e) $x$ does not satisfy $(*)_{(-f)}$.

Proof. The set $g r f$ being closed in $H \times \mathbb{R}$, we can use the symmetry of $T_{g r f}^{D}(x, f(x))$ for all $(x, f(x))$ in a residual set of $g r f$, hence for all $x$ in a residual set of $H$. Moreover, we remark that $(0,-1)$ belongs to $T_{g r f}^{D}(x, f(x))$ if and only if $x$ satisfies $(*)_{f}$. 
One can ask the following questions:

1. How often can $(*)_{f}$ (and/or $\left.(*)_{(-f)}\right)$ hold for a locally Lipschitz function $f$ on a separable Hilbert space $H$ ?

2. Can we find a countable orthonormal basis $\left\{e_{n}\right\}$ of $H$ such that the intersection of the $\left\{e_{n}\right\}$-full set of Theorem 6.1 with the set of points where $(*)_{f}\left(\right.$ and/or $\left.(*)_{(-f)}\right)$ does not hold, is a dense subset of $H$ ?

The answer to the second question is not known, but an affirmative one would yield another proof of Preiss's Theorem [21] in the setting of a separable Hilbert space. As for the first question, we know that for a lower semicontinuous real-valued function $f$ on a Hilbert space, the D-subdifferential is nonempty [9] on a dense set of $H$, implying that a dense set of points of $H$ do not satisfy $(*)_{f}$. However, $(*)_{f}$ seems to be a stronger property than that of the emptiness of the D-subdifferential, and we could be tempted to believe that $(*)_{f}$ could hold only in a set of first category. As the next proposition shows, this is not the case:

Proposition 6.6. Let $H$ be a real separable Hilbert space. Then there exists a locally Lipschitz function $f$ which is Gâteaux differentiable everywhere on $H$, but such that the set $\left\{x \in H: f_{D}^{\prime}(x ; 0)=0\right\}$ is not a residual set of $H$.

Proof. Let $f$ be the function in Preiss's famous counterexample (see [22] and [27]). It can be shown that $f$ is a locally Lipschitz Gâteaux differentiable function on $H$ that is not Fréchet differentiable on a residual set of $H$. Suppose that $f_{D}^{\prime}(x ; 0)=0$ for all $x$ in a residual set of $H$; then by Proposition 6.5 , we would have $( \pm f)_{D}^{\prime}(x ; 0)=$ 0 for all $x$ in a residual set of $H$. Hence by Theorem 6.3, we would get the Fréchet differentiability of $f$ on a residual set, which is a contradiction. Actually for any locally Lipschitz function which is generically Gâteaux differentiable but not Fréchet differentiable on a residual set of $H$, we deduce that the set $\left\{x \in H: f_{D}^{\prime}(x ; 0)=0\right\}$ is not a residual set of $H$.

\section{Convex Functions, Pointwise Maxima and Distance Functions}

We will now consider special classes of functions for which Theorems 6.1 and 6.3 can be applied. To begin with, if $f$ is a convex continuous function on a separable Hilbert space $H$, then $f$ is locally Lipschitz and there exists an element $\xi_{x}$ in $H$ such that

$$
f(y)-f(x) \geq\left\langle\xi_{x}, y-x\right\rangle \quad \forall y \in H
$$

(see [7] and [19]). Hence, no point of $H$ has property $(*)_{f}$. So, by Proposition 6.5, for all $x$ in a residual set of $H$, we have $( \pm f)_{D}^{\prime}(x ; 0)=0$. Therefore, it is a simple application of Corollary 5.7 and Theorem 6.3 to derive the following well-known result (see [19] for another proof):

Theorem 7.1. Let $H$ be a real separable Hilbert space. Let $f: H \longrightarrow \mathbb{R}$ be a convex continuous function on $H$. Then for all $x$ in a residual set of $H, f_{F}^{\prime}(x)$ exists.

Let us now consider pointwise maxima. To be more precise, let us define:

Definition 7.1. Let $T$ be an index set. For all $t$ in $T$, let $f_{t}(\cdot)$ be a locally Lipschitz function on $H$ such that $\left(f_{t}\right)_{D}^{\prime}(x ; 0)=0$ for all $x$ in $H$ (as is the case when $f_{t}$ is convex or Fréchet-differentiable on $H$ ). Assume the function defined by $f(x):=$ $\max _{t \in T} f_{t}(x)$ is well-defined and locally Lipschitz on $H$. Then $f$ is called a regular pointwise-maximum function. 
Theorem 7.2. Let $\left\{e_{1}, e_{2}, \ldots\right\}$ be a countable orthonormal basis of $H$. Let $f$ be a regular pointwise-maximum function on $H$. Then for all $x$ in a $\left\{e_{n}\right\}$-full set of $H$, we have:

(a) $f_{G}^{\prime}(x)$ exists.

(b) $\partial_{D} f(x)=\left\{f_{G}^{\prime}(x)\right\}$.

The first assertion is an immediate consequence of Theorem 5.1, but the second one is new and is an easy consequence of Theorem 6.1 , since no point $x$ of $H$ satisfies property $(*)_{f}$.

Theorem 7.3. Let $H$ be a real separable Hilbert space. Let $f$ be a regular upper envelope of a finite family. Then $f$ is Fréchet differentiable on a residual set of $H$.

Proof. By Corollary 5.4, we know that $f_{G}^{\prime}(x)$ exists for all $x$ on a staunch set of $H$. Since $f_{D}^{\prime}(x ; 0)=0$ for all $x$ in $H$ (since no point $x$ of $H$ satisfies property $(*)_{f}$ ), the result follows easily from Theorem 6.3 and Proposition 6.5.

Finally, let us consider distance functions:

Definition 7.2. A set $F$ in $H$ is said to be proximinal if for all $x \notin F$, there exists an element $y_{x} \in F$ such that $\left\|x-y_{x}\right\|=d_{F}(x)$.

The following proposition gives examples of proximinal sets:

Proposition 7.4 (see [8]). (a) Any closed set in $\mathbb{R}^{n}$ is proximinal.

(b) Any closed and convex set in $H$ is proximinal. Moreover, the infimum defining the distance function associated with such a set is uniquely attained.

(c) Any weakly closed set in $H$ is proximinal.

Proposition 7.5. Let $F$ be a proximinal set in $H$. Then for all $x \notin F$, we have $\left(-d_{F}\right)_{D}^{\prime}(x ; 0)=0$.

Proof. Fix $x \notin F$. Let $\left\{t_{i}\right\}$ be a real positive sequence decreasing to 0 and let $\left\{v_{i}\right\}$ be a sequence in $H$ converging weakly to 0 such that

$$
\lim _{i \rightarrow+\infty} \frac{-d_{F}\left(x+t_{i} v_{i}\right)-\left(-d_{F}(x)\right)}{t_{i}} \text { exists. }
$$

We need to prove that this limit is nonnegative. Since $F$ is proximinal, there exists $y_{x}$ in $F$ such that $d_{F}(x)=\left\|x-y_{x}\right\|$. Moreover, for all $i=1,2, \ldots$, we have $d_{F}\left(x+t_{i} v_{i}\right) \leq\left\|x+t_{i} v_{i}-y_{x}\right\|$. Hence, for all $i=1,2, \ldots$, we obtain:

$$
\frac{-d_{F}\left(x+t_{i} v_{i}\right)-\left(-d_{F}(x)\right)}{t_{i}} \geq \frac{-\left\|x+t_{i} v_{i}-y_{x}\right\|-\left(-\left\|x-y_{x}\right\|\right)}{t_{i}} .
$$

Now, the function $z \longmapsto f(z):=-\left\|z-y_{x}\right\|$ is Fréchet differentiable at every $z \neq y_{x}$, in particular at $x$. Hence:

$$
\begin{aligned}
\lim _{i \rightarrow+\infty} \frac{-d_{F}\left(x+t_{i} v_{i}\right)-\left(-d_{F}(x)\right)}{t_{i}} & \geq \lim _{i \rightarrow+\infty} \frac{f\left(x+t_{i} v_{i}\right)-f(x)}{t_{i}} \\
& =\left\langle f_{F}^{\prime}(x), 0\right\rangle \\
& =0,
\end{aligned}
$$

as desired.

One can now deduce very easily the following known result (see [13] for an even stronger result): 
Theorem 7.6. Let $H$ be a real separable Hilbert space. Let $F$ be a closed proximinal set in $H$. Then on a residual set of $H \backslash F$, the function $d_{F}$ is Fréchet differentiable.

Proof. Note that $d_{F}$ is not necessarily convex; hence this is not a direct application of Theorem 7.1. But $F$ being closed in $H$, the function $\left(-d_{F}\right)$ is Gâteaux differentiable on a staunch set of $H \backslash F$ by Corollary 5.5. Now by Propositions 6.5 and 7.5, we get that $\left( \pm d_{F}\right)_{D}^{\prime}(x ; 0)=0$ on a residual set of $H \backslash F$, which implies the result by means of Theorem 6.3 .

When $F$ is convex, the function $d_{F}$ is known to be convex; hence it is Fréchet differentiable on a residual set of $H$ (by Theorem 7.1). But an extra hypothesis on $F$ gives the Fréchet differentiability of $d_{F}$ on a staunch set, as we now see:

Theorem 7.7. Let $H$ be a real separable Hilbert space. Let $F$ be a closed and convex set in $H$. Then on a staunch set of $H \backslash F$, the function $d_{F}$ is Fréchet differentiable.

Proof. The set $F$ being proximinal (Proposition 7.4 ), we have $\left(-d_{F}\right)(x ; 0)=0$ for all $x$ in $H \backslash F$ (Proposition 7.5). Also $F$ being convex, the function $d_{F}$ is Gâteaux differentiable on a staunch set of $H$ (Corollary 5.7) and $d_{F}(x ; 0)=0$ for all $x$ in $H$. Theorem 6.3 now gives the result.

\section{REFERENCES}

[1] Aronszajn N., Differentiability of Lipschitzian mappings between Banach spaces, Studia Math. 57 (1976), 147-190. MR 54:13562

[2] Bessis D.N., Analyse contingentielle et sousdifférentielle, PhD thesis, Université de Montréal, 1997.

[3] Borwein J.M., Strójwas H.M., Proximal analysis and boundaries of closed sets in Banach space. II. Applications, Canad. J. Math. 39 (1987), no. 2, 428-472. MR 88f:46034

[4] Bruckner A., Differentiation of real functions, Second edition, CRM Monograph Series, 1994. MR 94m:26001

[5] Choquet G., Outils topologiques et métriques de l'analyse mathématique, Cours rédigé par C. Mayer, Centre de Documentation Universitaire, Paris, 1969. MR 41:7033

[6] Clarke F.H., Generalized gradients and applications, Trans. Amer. Math. Soc. 205 (1975), 247-262. MR 51:3373

[7] Clarke F.H., Optimization and nonsmooth analysis, Second edition, Classics in Applied Mathematics 5, Society for Industrial and Applied Mathematics (SIAM), Philadelphia, 1990. MR 91e: 49001

[8] Clarke F.H., Ledyaev Yu.S., Stern R.J., Wolenski P.R., Nonsmooth analysis and optimal control, to be published in Graduate Texts in Mathematics, Springer-Verlag. CMP 98:06

[9] Clarke F.H., Ledyaev Yu.S., Wolenski P.R., Proximal analysis and minimization principles, J. Math. Anal. Appl. 196 (1995), 722-735. MR 96i:49028

[10] Dolženko E.P., Boundary properties of arbitrary functions (Russian), Izv. Akad. Nauk SSSR Ser. Mat. 31 (1967), 3-14. MR 36:388

[11] Fabián M., Preiss D., On intermediate differentiability of Lipschitz functions on certain Banach spaces, Proc. Amer. Math. Soc. 113 (1991), no. 3, 733-740. MR 92g:46048

[12] Halmos P.R., Measure Theory, D.Van Nostrand Company, Inc., New York, 1986. MR 11:504d

[13] Giles J.R., Sciffer S., Locally Lipschitz functions are generically pseudo-regular on separable Banach spaces, Bull. Austral. Math. Soc. 47 (1993), 205-212. MR 94a:58022

[14] Kuratowski K., Topology, New edition, revised and augmented, Academic Press, New YorkLondon, 1966. MR 36:840

[15] Lukeš J., Malý J., Zajícek L., Fine topology methods in real analysis and potential theory, Lecture Notes in Mathematics, 1189, Springer-Verlag, Berlin-New York, 1986. MR 89b:31001

[16] Mignot F., Contrôle dans les inéquations variationnelles elliptiques, J. Funct. Anal. 22 (1976), 130-185. MR 54:11136 
[17] de Mises M.R., La base géométrique du théorème de M. Mandelbrojt sur les points singuliers d'une fonction analytique, C.R. Acad. Sci. Paris Sér. I Math. 205 (1937), 1353-1355.

[18] Nekvinda A., Zaj̃̄cek L., A simple proof of Rademacher Theorem, Časopis Pěst. Mat. 113 (1988), no. 4, 337-341. MR 90c:26043

[19] Phelps R.R., Convex functions, monotone operators and differentiability, Second edition, Lecture Notes in Mathematics, 1364, Springer-Verlag, Berlin, 1993. MR 94f:46055

[20] Phelps R.R., Gaussian null sets and differentiability of Lipschitz map on Banach spaces, Pacific J. Math. 77 (1978), no. 2, 523-531. MR 80m:46040

[21] Preiss D., Differentiability of Lipschitz functions on Banach spaces, J. Funct. Anal. 91 (1990), 312-345. MR 91g:46051

[22] Preiss D., Gâteaux differentiable Lipschitz functions need not be Fréchet differentiable on a residual subset, Rend. Circ. Mat. Palermo Suppl. 2 (1982), 217-222. MR 84h:46054

[23] Rademacher H., Über partielle und totale Differenzierbarkeit I., Math. Ann. 89 (1919), 340359.

[24] Saint-Pierre J., Sur le théorème de Rademacher, Travaux Sém. Anal. Convexe 12 (1982), exp. no. 2, 2.1-2.10. MR 84f:26016

[25] Saks S., Theory of the integral, Second revised edition, Dover Publications, Inc., New York, 1964. MR 29:4850

[26] Shu Zhong S., Choquet theorem and nonsmooth analysis, J. Math. Pures Appl. 67 (1988), no. 4, 411-432. MR 90e:58008

[27] Shu Zhong S., Différentiabilité d'une fonction localement lipschitzienne dans un espace de Banach séparable, J. Systems Sci. Math. Sci. 3 (1983), no. 2, 112-119. MR 88i:58013

[28] Zajícek L., Differentiability of the distance function and points of multivaluedness of the metric projection in Banach space, Czechoslovak Math. J. 33(108) (1983), no. 2, 292-308. MR 85b: 46025

[29] Zaj̃icek L., Sets of $\sigma$-porosity and sets of $\sigma$-porosity (q), Časopis Pěst. Mat. 101 (1976), no. 4, 350-359. MR 56:15935

Centre for Process Systems Engineering, Imperial College of Science, Technology and Medicine, Exhibition Road, London, United Kingdom, SW7 2AZ

E-mail address: d.bessis@ic.ac.uk

Mathématiques, Université de Lyon I, 69622 Villeurbanne, France, and Centre de Recherches Mathématiques, Université de Montréal, C. P. 6128, Succ. Centre-ville, Montréal, Québec, Canada, H3C 3J7

E-mail address: clarke@crm.umontreal.ca 